\title{
ON IMPLICIT VARIABLES IN OPTIMIZATION THEORY
}

\author{
Matúš Benko * Patrick Mehlitz ${ }^{\dagger}$
}

\begin{abstract}
Implicit variables of a mathematical program are variables which do not need to be optimized but are used to model feasibility conditions. They frequently appear in several different problem classes of optimization theory comprising bilevel programming, evaluated multiobjective optimization, or nonlinear optimization problems with slack variables. In order to deal with implicit variables, they are often interpreted as explicit ones. Here, we first point out that this is a lightheaded approach which induces artificial locally optimal solutions. Afterwards, we derive various Mordukhovich-stationarity-type necessary optimality conditions which correspond to treating the implicit variables as explicit ones on the one hand, or using them only implicitly to model the constraints on the other. A detailed comparison of the obtained stationarity conditions as well as the associated underlying constraint qualifications will be provided. Overall, we proceed in a fairly general setting relying on modern tools of variational analysis. Finally, we apply our findings to different well-known problem classes of mathematical optimization in order to visualize the obtained theory.
\end{abstract}

Keywords: Implicit variables, Local minimizers, Metric subregularity, M-stationarity, Variational analysis

$\operatorname{MSC}(2010): 49 \mathrm{~J} 53,90 \mathrm{C}_{30}, 90 \mathrm{C} 33$

\section{INTRODUCTION}

In this paper, we consider the mathematical program

$$
\begin{aligned}
f(z) & \rightarrow \min _{z} \\
0 & \in H(z) \\
z & \in M
\end{aligned}
$$

where $f: \mathbb{R}^{n} \rightarrow \mathbb{R}$ is a given locally Lipschitz continuous objective function and the set-valued mapping $H: \mathbb{R}^{n} \rightrightarrows \mathbb{R}^{s}$ attains the form

$$
H(z):=\bigcup_{\lambda \in F(z)} G(z, \lambda)
$$

for set-valued mappings $F: \mathbb{R}^{n} \rightrightarrows \mathbb{R}^{m}$ and $G: \mathbb{R}^{n} \times \mathbb{R}^{m} \rightrightarrows \mathbb{R}^{s}$ which possess a closed graph, respectively. Furthermore, $M \subset \mathbb{R}^{n}$ is a nonempty and closed set of simple structure, e.g., a typical constraint set

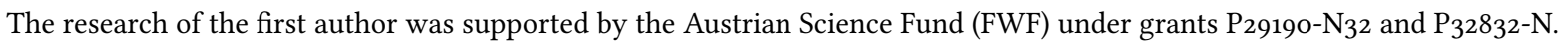
Moreover, the first author would like to thank the Institute of Computational Mathematics, Johannes Kepler University Linz, for access to the infrastructure of the institute.

* Applied Mathematics and Optimization. University of Vienna, Austria. matus.benko@univie.ac.at. ORCID: oooo-ooo33307-7939.

† Institute of Mathematics, Chair of Optimal Control. Brandenburgische Technische Universität Cottbus-Senftenberg, Germany. mehlitz@b-tu.de. ORCID: oooo-ooo2-9355-850X. 
defined via standard inequality and equality constraints satisfying a suitable constraint qualification. The feasible set of $(\mathrm{P})$ will be denoted by $Z \subset \mathbb{R}^{n}$. We emphasize that the objective in (P) is only minimized w.r.t. $z$ and not w.r.t. $\lambda$ although the latter variable is used implicitly in order to model $Z$. That is why we call $\lambda$ an implicit variable. Let us note that the rather general model (P) covers several problem classes from optimization theory, which are modeled with the aid of implicit variables, e.g., bilevel programming problems, evaluated multiobjective programs, or programs with slack variables, see Section 2 as well. In these examples, the implicit variables are given by means of the lower level Lagrange multipliers, scalarization parameters, or the introduced slack variables, respectively.

The main difficulty in $(\mathrm{P})$ is the appearing union of image sets associated with the given set-valued mappings $F$ and $G$. An easy approach which can be used to handle this issue is to interpret $\lambda$ as a meaningful variable. This leads to the consideration of

$$
\begin{aligned}
f(z) & \rightarrow \min _{z, \lambda} \\
\lambda & \in F(z) \\
0 & \in G(z, \lambda) \\
z & \in M
\end{aligned}
$$

where $\lambda$ now takes the role of an explicit variable. We denote the feasible set of this program by $\tilde{Z} \subset \mathbb{R}^{n} \times \mathbb{R}^{m}$. As we will see in Section 4.1, there is a one-to-one correspondence between the global minimizers of $(\mathrm{P})$ and $(\mathrm{Q})$. However, the relationship between local minimizers is far more delicate. Particularly, there may exist local minimizers of (Q) which do not correspond to local minimizers of (P). Similar issues are pointed out in the context of bilevel programming, see [2, 20, 22, 23, 43], w.r.t. the use of slack variables in logical programming, see [44, 46], or cardinality-constrained optimization, see [17], and will be generalized in this paper.

Let us briefly mention that the concept of implicit variables can be extended to related areas of optimization theory like the numerical solution of generalized equations resulting from optimality conditions of convex optimization programs. Here, it is also common to introduce intermediate or slack variables in order to state the convex chain rule for compositions, see e.g. [15, Section 4], or to decompose the inclusion to make it more tractable, see [35, Section 5]. It sometimes may be beneficial, however, to formulate the assumptions ensuring convergence in the implicit-variable-free setting.

Observing that the computation of local minimizers of (Q) does not generally yield local minimizers of $(\mathrm{P})$ while noting that $(\mathrm{Q})$ is likely to be a nonconvex program due to the underlying applications we have in mind, a direct treatment of $(\mathrm{P})$ seems to be reasonable. With the aid of modern variational analysis, in particular limiting coderivatives of set-valued mappings, see $[48,53]$, we will infer necessary optimality conditions of Mordukhovich-stationarity-type (subsequently, we will use the term M-stationarity for brevity) for $(\mathrm{P})$ under mild constraint qualifications. More precisely, we first derive optimality conditions comprising the implicitly known coderivative of the mapping $H$ under metric subregularity of $H$. Afterwards, we study assumptions which allow us to state these optimality conditions in terms of the coderivatives associated with $F$ and $G$. Clearly, this makes the utilization of a coderivative chain rule necessary, so we discuss weak conditions ensuring its applicability. Therefore, we make use of the recent results from $[6,9]$ which are mainly based on the presence of metric subregularity for so-called "feasibility" mappings and inner semicompactness for so-called "intermediate" mappings. Interestingly, these assumptions are mainly inherent for the example problems from Section 2 which underlines the power of this approach. On the other hand, using problem (Q) as the starting point enables us to avoid the (coderivative of) the mapping $H$. A similar role is then played by an auxiliary mapping, which depends on both, $z$ and $\lambda$. As it will turn out, the procedures sketched above lead to three stationarity systems and diverse constraint qualifications which differ from each other w.r.t. their degree of explicitness. We will provide a detailed comparison of all these stationarity conditions and constraint qualifications, and we will comment on their respective relation to the problem (Q). In 
[1], the authors discuss related issues by means of an equilibrium-constrained mathematical problem with lower level inequality constraints. It turns out that there is a significant difference between the M-stationarity conditions for problem (P), which are formulated without taking into account the special structure of $H$ and, hence, without the variable $\lambda$, and the M-stationarity conditions of (Q). Moreover, the difference between the corresponding constraint qualifications, i.e., the conditions that guarantee the validity of the two types of M-stationarity at local minimizers, is even more striking. Here, we generalize and deepen the approach from [1]. Let us briefly note that the theory of this paper can be extended directly to the situation where $\mathbb{R}^{n}, \mathbb{R}^{m}$, and $\mathbb{R}^{s}$ are replaced by arbitrary Euclidean spaces. For simplicity, however, we focus on the most elementary setting.

The remaining parts of this paper are organized as follows: In Section 2, we present three prototypical example classes of optimization problems where implicit variables are used to model the feasible set, namely bilevel optimization problems, evaluated multiobjective optimization problems, and mathematical programs with cardinality constraints. We provide an overview of the notation used in this manuscript before recalling the definitions and some background information about the fundamental tools of variational analysis we are going to exploit here in Section 3. Furthermore, we motivate a rather abstract notion of M-stationarity for mathematical problems with generalized equation constraints and provide some calculus rules for the coderivative of compositions and products of set-valued mappings. Afterwards, Section 4 is dedicated to the abstract analysis of the program $(\mathrm{P})$ via $(\mathrm{Q})$. First, we study the relationship between the solutions of $(\mathrm{P})$ and $(\mathrm{Q})$ in Section 4.1. Second, we derive three potentially distinct notions of M-stationarity for $(\mathrm{P})$ as well as associated constraint qualifications in Section 4.2. Furthermore, we clarify how to obtain explicit M-stationarity conditions in terms of initial problem data from the abstract $\mathrm{M}$-stationarity conditions of $(\mathrm{P})$. As we will demonstrate in Section 4.3, the necessary assumptions are inherently satisfied in many practically relevant settings. Finally, we briefly comment on the sufficiency of the introduced $\mathrm{M}$-stationarity notions in the presence of convexity in Section 4.4. In Section 5, we apply some of our findings to the aforementioned example problems. As it will turn out, we recover or even enhance available results from the literature with our approach. Finally, we present some concluding remarks in Section 6.

\section{MOTIVATING EXAMPLES}

In this section, we present three prototypical classes of optimization problems whose respective feasible sets can be modeled with the aid of implicit variables.

\subsection{BILEVEL PROGRAMMING}

Quite often, hierarchical decision making appears naturally in real world problems raising in economics, logistics, natural sciences, or engineering. In case where two decision makers are involved, so-called bilevel optimization problems can be used to model and study the underlying applications theoretically and numerically, see $[4,19,21,48,54]$. For given parameters $x \in \mathbb{R}^{n_{1}}$, let us consider the parametric optimization problem

$$
\begin{gathered}
j(x, y) \rightarrow \min _{y} \\
g(y) \in C
\end{gathered}
$$

where $j: \mathbb{R}^{n_{1}} \times \mathbb{R}^{n_{2}} \rightarrow \mathbb{R}$ is twice continuously differentiable and convex w.r.t. $y$ for each $x \in \mathbb{R}^{n_{1}}$ while $g: \mathbb{R}^{n_{2}} \rightarrow \mathbb{R}^{m}$ is twice continuously differentiable and convex w.r.t. the closed, convex cone $C \subset \mathbb{R}^{m}$. Let us set $\Gamma:=\left\{y \in \mathbb{R}^{n_{2}} \mid g(y) \in C\right\}$. One generally refers to (LL) as the lower level or follower's problem. It is well known that due to the convexity of $j(x, \cdot)$ and $\Gamma$, the set of optimal solutions associated with 
(LL) is equivalently characterized by

$$
-\nabla_{y} j(x, y) \in \widehat{\mathcal{N}}_{\Gamma}(y) .
$$

Under a suitable constraint qualification, we can characterize the set of optimal solutions associated with (LL) equivalently by the generalized equation

$$
-\nabla_{y} j(x, y) \in \nabla g(y)^{\top} \widehat{\mathcal{N}}_{C}(g(y)) .
$$

Above, we exploited the notion of the regular normal cone which coincides with the normal cone of convex analysis due to convexity of $\Gamma$ and $C$, see Section 3 .

The superordinate so-called upper level or leader's problem, given by

$$
\begin{aligned}
f(x, y) & \rightarrow \min _{x, y} \\
x & \in S \\
y & \in \Psi(x),
\end{aligned}
$$

where $S \subset \mathbb{R}^{n_{1}}$ is a closed set and $\Psi: \mathbb{R}^{n_{1}} \rightrightarrows \mathbb{R}^{n_{2}}$ is the solution mapping associated with (LL), is equivalent to

$$
\begin{aligned}
f(x, y) & \rightarrow \min _{x, y} \\
x & \in S \\
0 & \in \bigcup_{\lambda \in \widehat{\mathcal{N}}_{C}(g(y))}\left\{\nabla_{y} j(x, y)+\nabla g(y)^{\top} \lambda\right\}
\end{aligned}
$$

whenever the aforementioned assumptions are valid. The latter is a program of type $(\mathrm{P})$ in the sense

$$
F(z):=\widehat{\mathcal{N}}_{C}(g(y)), \quad G(z, \lambda):=\nabla_{y} j(x, y)+\nabla g(y)^{\top} \lambda, \quad M:=S \times \mathbb{R}^{n_{2}}
$$

where we used $z:=(x, y)$ and $n:=n_{1}+n_{2}$. Particularly, the implicit variable $\lambda$ is the Lagrange multiplier associated with the constraints in (LL). It is well known that interpreting $\lambda$ as a variable in (BPP) may cause difficulties w.r.t. local minimizers, see e.g. [2, 20, 22, 43]. The authors in [1], where the particular setting $C:=\mathbb{R}_{-}^{m}$ is discussed, focused on a qualitative comparison of the reformulation

$$
\begin{aligned}
f(x, y) & \rightarrow \min _{x, y} \\
x & \in S \\
-\nabla_{y} j(x, y) & \in \widehat{\mathcal{N}}_{\Gamma}(y),
\end{aligned}
$$

which is fully equivalent to the original bilevel programming problem, and the problem $(\mathrm{Q})$ associated with (BPP) regarding constraint qualifications and M-stationarity-type optimality conditions. They came up with the observation that the respective stationarity systems as well as constraint qualifications differ significantly. The analysis in this paper will put their results into a more general context.

\subsection{EVALUATING WEAKLY EFFICIENT POINTS IN MULTICRITERIA OPTIMIZATION}

For a twice continuously differentiable componentwise convex function $j: \mathbb{R}^{n} \rightarrow \mathbb{R}^{m}$ with $m \geq 2$ components and a nonempty, closed, convex set $\Gamma \subset \mathbb{R}^{n}$, we consider the following multicriteria optimization problem which is a standard model from vector optimization, see [26, 41]:

(MOP)

$$
\begin{aligned}
j(z) & \rightarrow \text { “min" } \\
z & \in \Gamma .
\end{aligned}
$$


The quotation marks emphasize that classical minimization is not possible since the componentwise natural ordering in $\mathbb{R}^{m}$ only provides a partial order which is not total. In order to overcome this problem, several different notions of so-called efficiency have been derived in order to characterize reasonable feasible points of (MOP). In this context, a point $\bar{z} \in \Gamma$ is called weakly efficient for (MOP) if the condition

$$
\left(j(\bar{z})-\mathbb{R}_{++}^{m}\right) \cap j(\Gamma)=\varnothing
$$

holds where we used $\mathbb{R}_{++}^{m}:=\left\{y \in \mathbb{R}^{m} \mid \forall i \in\{1, \ldots, m\}: y_{i}>0\right\}$. Let $\Gamma_{\text {we }} \subset \mathbb{R}^{n}$ be the set of weakly efficient points associated with (MOP). Using a classical linear scalarization approach, see [26], one obtains the characterization

$$
\bar{z} \in \Gamma_{\mathrm{we}} \quad \Longleftrightarrow \quad \exists \lambda \in \Delta \forall z \in \Gamma: \lambda^{\top} j(\bar{z}) \leq \lambda^{\top} j(z)
$$

of weak efficiency since the problem data in (MOP) is convex. Above, $\Delta \subset \mathbb{R}^{m}$ given by

$$
\Delta:=\left\{\lambda \in \mathbb{R}^{m} \mid \lambda \geq 0, \sum_{i=1}^{m} \lambda_{i}=1\right\}
$$

denotes the standard simplex. In practice, the set $\Gamma_{\mathrm{we}}$ will be quite large in general which is why it is desirable to shrink it for applicability purposes using another decision criterion given in form of a scalar function, see e.g. [10, 12, 13, 39]. Here, we investigate the evaluated multiobjective optimization problem

$$
\begin{gathered}
f(z) \rightarrow \min \\
z \in \Gamma_{\mathrm{we}} .
\end{gathered}
$$

Invoking the above arguments, this is equivalent to

$$
\begin{aligned}
f(z) & \rightarrow \min \\
0 & \in \bigcup_{\lambda \in \Delta} \Psi(\lambda)-z
\end{aligned}
$$

where $\Psi: \mathbb{R}^{m} \rightrightarrows \mathbb{R}^{n}$ is the set-valued mapping defined by

$$
\Psi(\lambda):= \begin{cases}\underset{z}{\operatorname{argmin}}\left\{\lambda^{\top} j(z) \mid z \in \Gamma\right\} & \lambda \in \Delta, \\ \varnothing & \lambda \notin \Delta .\end{cases}
$$

Obviously, this is a program of type $(\mathrm{P})$ where the scalarization parameter takes the role of the implicit variable. Particularly, the data from $(\mathrm{P})$ takes the form

$$
F(z):=\Delta, \quad G(z, \lambda):=\Psi(\lambda)-z, \quad M:=\mathbb{R}^{n} .
$$

It has been reported in [23] that interpreting scalarization parameters as variables is generally delicate in multiobjective bilevel programming, and this observation clearly extends to (EMOP) as well.

\subsection{CARDINALITY-CONSTRAINED PROGRAMMING}

For some vector $z \in \mathbb{R}^{n}$, let $\|z\|_{0}$ denote the number of non-zero entries of $z$. In order to guarantee sparsity of solutions to optimization problems, one may consider the so-called cardinality-constrained mathematical program

$$
\begin{aligned}
f(z) & \rightarrow \min \\
z & \in M \\
\|z\|_{0} & \leq \kappa
\end{aligned}
$$


where $\kappa \in \mathbb{N}$ satisfies $1 \leq \kappa \leq n-1$, see e.g. [11, 16, 17, 18, 45,50,51] for an overview of existing reformulations, optimality conditions, solution algorithms, and further references.

Let us rewrite the cardinality constraint $\|z\|_{0} \leq \kappa$ as a constraint of type $q(z) \in D$ where $q: \mathbb{R}^{n} \rightarrow \mathbb{R}^{\ell}$ is continuously differentiable and $D \subset \mathbb{R}^{\ell}$ is the union of finitely many convex polyhedral sets, allowing us to interpret (CCMP) as a so-called disjunctive optimization problem, see e.g. [7, 28, 45]. Therefore, we introduce $\mathcal{J}_{\kappa}:=\left\{\alpha \in\{0,1\}^{n} \mid \sum_{i=1}^{n} \alpha_{i}=\kappa\right\}$. Furthermore, for each $\alpha \in\{0,1\}^{n}$, we set

$$
\mathbb{R}_{\alpha}^{n}:=\operatorname{span}\left\{\mathrm{e}_{i} \mid i=1, \ldots, n, \alpha_{i}=1\right\}
$$

where $\mathrm{e}_{i} \in \mathbb{R}^{n}$ denotes the $i$-th unit vector in $\mathbb{R}^{n}$. Finally, we set $D_{\kappa}:=\bigcup_{\alpha \in \mathcal{J}_{\kappa}} \mathbb{R}_{\alpha}^{n}$. Then we easily see

$$
\forall z \in \mathbb{R}^{n}: \quad\|z\|_{0} \leq \kappa \Longleftrightarrow z \in D_{\kappa} .
$$

The variational geometry of $D_{\kappa}$ has been explored in [50, 51]. Particularly, exploiting the limiting and regular normal cone to $D_{\kappa}$, reasonable notions of M- and strong stationarity are available for (CCMP).

It has been observed in [17, Theorem 3.2] that we have

$$
\forall z \in \mathbb{R}^{n}: \quad\|z\|_{0} \leq \kappa \Longleftrightarrow \exists \lambda \in \mathbb{R}^{n}: \mathrm{e}^{\top} \lambda \geq n-\kappa,\left(z_{i}, \lambda_{i}\right) \in C \forall i \in\{1, \ldots, n\}
$$

where we used

$$
C:=\left\{(a, b) \in \mathbb{R}^{2} \mid a b=0, b \in[0,1]\right\},
$$

and that gave rise to the reformulation of (CCMP) as

$$
\begin{aligned}
f(z) & \rightarrow \min _{z, \lambda} \\
z & \in M \\
\mathrm{e}^{\top} \lambda & \geq n-\kappa \\
\left(z_{i}, \lambda_{i}\right) & \in C \quad i=1, \ldots, n .
\end{aligned}
$$

Above, $\mathrm{e} \in \mathbb{R}^{n}$ denotes the all-ones vector. Clearly, the variable $\lambda$ plays the role of an explicit variable in (2.4) although it is not relevant for the purpose of minimization. In order to state (2.4) in the form $(\mathrm{P})$, one may choose

$$
F(z) \equiv\left\{\lambda \in \mathbb{R}^{n} \mid \mathrm{e}^{\top} \lambda \geq n-\kappa\right\}, \quad G(z, \lambda):=\prod_{i=1}^{n}\left(C-\left(z_{i}, \lambda_{i}\right)\right),
$$

i.e., $s=2 n$ holds in this particular case.

It has been reported in [17] that (CCMP) and (2.4) are equivalent w.r.t. global minimizers, that the local minimizers of (CCMP) can be found among the local minimizers of (2.4), and that for a local minimizer $(\bar{z}, \bar{\lambda}) \in \mathbb{R}^{n} \times \mathbb{R}^{n}$ of (2.4), which satisfies $\|\bar{z}\|_{0}=\kappa, \bar{z}$ is a local minimizer of (CCMP). The situation where $\|\bar{z}\|_{0}<\kappa$ holds, however, has been shown to be crucial since, in this case, $\bar{z}$ does not need to be locally optimal for (CCMP). First- and second-order optimality conditions for (CCMP) via its surrogate (2.4) have been derived in $[16,17,18]$ while the authors in $[45,50,51]$ exploited $(2.3)$ in order to infer optimality conditions for (CCMP) without relying on implicit variables. To the best of our knowledge, a detailed comparison of both approaches does not exist in the literature. Some regarding remarks, however, can be found in the papers $[16,45]$. We also refer the interested reader to [5] where yet another approach to cardinality-constrained optimization is discussed which also avoids the appearance of implicit variables.

\section{NOTATION AND PRELIMINARIES}

\subsection{NOTATION}

Throughout the manuscript, we make use of the standard concepts of variational analysis, see $[48,53]$. 


\section{BASIC NOTATION}

In this manuscript, we equip $\mathbb{R}^{n}$ with the common Euclidean inner product and the common Euclidean norm $\|\cdot\|$. In order to extend the notion of norms to product spaces, we use the sum of the underlying Euclidean norms to induce a norm in the product space. Due to notational purposes, for arbitrary $z \in \mathbb{R}^{n}$ and $w \in \mathbb{R}^{m}$, we use both notations

$$
\left(\begin{array}{c}
z \\
w
\end{array}\right) \quad \text { and } \quad(z, w)
$$

in order to represent elements of $\mathbb{R}^{n} \times \mathbb{R}^{m}$. We use $\mathbb{B}$ in order to denote the closed unit ball around 0 . For a set $A \subset \mathbb{R}^{n}$ and $z \in \mathbb{R}^{n}$, we exploit $z+A=A+z:=\{z+a \mid a \in A\}$ for brevity. Furthermore, we use

$$
A^{\circ}:=\left\{\xi \in \mathbb{R}^{n} \mid \forall a \in A: \xi^{\top} a \leq 0\right\}, \quad A^{\perp}:=\left\{\xi \in \mathbb{R}^{n} \mid \forall a \in A: \xi^{\top} a=0\right\}
$$

in order to represent the polar cone and the annihilator of $A$. We make use of $z^{\perp}:=\{z\}^{\perp}$. We set

$$
\operatorname{dist}(z, A):=\inf \{\|a-z\| \mid a \in A\}
$$

in order to denote the distance of $z$ to $A$. Throughout the manuscript, we exploit $\mathbb{R}_{+}:=\{s \in \mathbb{R} \mid s \geq 0\}$ and $\mathbb{R}_{-}:=\{s \in \mathbb{R} \mid s \leq 0\}$.

\section{SET-VALUED MAPPINGS}

For a set-valued mapping $\Upsilon: \mathbb{R}^{n} \rightrightarrows \mathbb{R}^{m}$, the sets gph $\Upsilon:=\left\{(z, w) \in \mathbb{R}^{n} \times \mathbb{R}^{m} \mid w \in \Upsilon(z)\right\}$, dom $\Upsilon:=$ $\left\{z \in \mathbb{R}^{n} \mid \Upsilon(z) \neq \varnothing\right\}$, rge $\Upsilon:=\left\{w \in \mathbb{R}^{m} \mid \exists z \in \mathbb{R}^{n}: w \in \Upsilon(z)\right\}$, and ker $\Upsilon:=\left\{z \in \mathbb{R}^{n} \mid 0 \in \Upsilon(z)\right\}$ are called graph, domain, range, and kernel of $\Upsilon$, respectively. The set-valued mapping $\Upsilon^{-1}: \mathbb{R}^{m} \rightrightarrows \mathbb{R}^{n}$, given by $\Upsilon^{-1}(w):=\left\{z \in \mathbb{R}^{n} \mid w \in \Upsilon(z)\right\}$ for all $w \in \mathbb{R}^{m}$, is referred to as the inverse of $\Upsilon$.

The mapping $\Upsilon$ is called locally bounded at $\bar{z} \in \operatorname{dom} \Upsilon$ whenever we find a neighborhood $U \subset \mathbb{R}^{n}$ of $\bar{z}$ and a bounded set $B \subset \mathbb{R}^{m}$ such that $\Upsilon(z) \subset B$ holds for all $z \in U$. Furthermore, $\Upsilon$ is said to be inner semicompact at $\bar{z}$ w.r.t. $\Omega \subset \mathbb{R}^{n}$ if for each sequence $\left\{z_{k}\right\}_{k \in \mathbb{N}} \subset \Omega$ such that $z_{k} \rightarrow \bar{z}$, there is a convergent sequence $\left\{w_{l}\right\}_{l \in \mathbb{N}} \subset \mathbb{R}^{m}$ and a subsequence $\left\{z_{k_{l}}\right\}_{l \in \mathbb{N}}$ such that $w_{l} \in \Upsilon\left(z_{k_{l}}\right)$ holds for all $l \in \mathbb{N}$. Clearly, whenever $\Upsilon$ is locally bounded at $\bar{z}$, it is inner semicompact w.r.t. dom $\Upsilon$ at this point. Similarly, $\Upsilon$ is called inner semicontinuous at $(\bar{z}, \bar{w}) \in \operatorname{gph} \Upsilon$ w.r.t. $\Omega$ if for each sequence $\left\{z_{k}\right\}_{k \in \mathbb{N}} \subset \Omega$ satisfying $z_{k} \rightarrow \bar{z}$, there exists a sequence $\left\{w_{k}\right\}_{k \in \mathbb{N}} \subset \mathbb{R}^{m}$ such that $w_{k} \rightarrow \bar{w}$ and $w_{k} \in \Upsilon\left(z_{k}\right)$ for all sufficiently large $k \in \mathbb{N}$.

We call $\Upsilon$ convex whenever the property

$$
\forall z_{1}, z_{2} \in \mathbb{R}^{n}, \forall \alpha \in[0,1]: \quad \alpha \Upsilon\left(z_{1}\right)+(1-\alpha) \Upsilon\left(z_{2}\right) \subset \Upsilon\left(\alpha z_{1}+(1-\alpha) z_{2}\right)
$$

holds, i.e., whenever gph $\Upsilon$ is convex. This property particularly holds for set-valued mappings of the form $\Upsilon(z):=g(z)-C, z \in \mathbb{R}^{n}$, where $C \subset \mathbb{R}^{m}$ is a closed, convex cone and the single-valued mapping $g: \mathbb{R}^{n} \rightarrow \mathbb{R}^{m}$ is convex w.r.t. $C$, i.e.,

$$
\forall z_{1}, z_{2} \in \mathbb{R}^{n}, \forall \alpha \in[0,1]: \quad g\left(\alpha z_{1}+(1-\alpha) z_{2}\right)-\alpha g\left(z_{1}\right)-(1-\alpha) g\left(z_{2}\right) \in C .
$$

Let us recall some essential Lipschitzian properties of set-valued mappings. Therefore, we fix a point $(\bar{z}, \bar{y}) \in$ gph $\Upsilon$. Recall that $\Upsilon$ possesses the Aubin property at $(\bar{z}, \bar{w})$ whenever there are a constant $\kappa>0$ and neighborhoods $U$ of $\bar{z}$ and $V$ of $\bar{w}$ such that

$$
\forall z_{1}, z_{2} \in U \forall w \in \Upsilon\left(z_{1}\right) \cap V: \quad \operatorname{dist}\left(w, \Upsilon\left(z_{2}\right)\right) \leq \kappa\left\|z_{1}-z_{2}\right\| .
$$


Furthermore, $\Upsilon$ is called metrically regular at $(\bar{z}, \bar{w})$ whenever there are $\kappa>0$ as well as neighborhoods $U$ and $V$ of $\bar{z}$ and $\bar{w}$, respectively, such that

$$
\forall z \in U, \forall w \in V: \quad \operatorname{dist}\left(z, \Upsilon^{-1}(w)\right) \leq \kappa \operatorname{dist}(w, \Upsilon(z)) .
$$

It is well known that $\Upsilon$ is metrically regular at $(\bar{z}, \bar{w})$ if and only if $\Upsilon^{-1}$ possesses the Aubin property at $(\bar{w}, \bar{z})$. Finally, recall that $\Upsilon$ is referred to as metrically subregular at $(\bar{z}, \bar{w})$ whenever there exist a constant $\kappa>0$ and a neighborhood $U$ of $\bar{z}$ satisfying

$$
\forall z \in U: \quad \operatorname{dist}\left(z, \Upsilon^{-1}(\bar{w})\right) \leq \kappa \operatorname{dist}(\bar{w}, \Upsilon(z)) .
$$

The infimum of all such constants $\kappa$ is referred to as the modulus of metric subregularity. Let us mention that $\Upsilon$ is metrically subregular at $(\bar{z}, \bar{w})$ if and only if the inverse $\Upsilon^{-1}$ is so-called calm at $(\bar{w}, \bar{z})$, see $[37,38]$ for a definition and further details. It has turned out that metric subregularity is of essential importance in order to guarantee applicability of the prominent calculus for the limiting constructions of generalized differentiation. This is one of the reasons why sufficient conditions for its presence were studied, see e.g. $[3,7,27,30,40,60]$ and the references therein as well as the aforementioned papers dealing with calmness. We would like to mention that each polyhedral set-valued mapping, i.e., a set-valued mapping whose graph is the union of finitely many convex polyhedral sets, is metrically subregular at each point of its graph. This result dates back to [52].

\section{VARIATIONAL ANALYSIS}

For a closed set $A \subset \mathbb{R}^{n}$ and some point $\bar{z} \in A$, we refer to

$$
\mathcal{T}_{A}(\bar{z}):=\left\{d \in \mathbb{R}^{n} \mid \exists\left\{z_{k}\right\}_{k \in \mathbb{N}} \subset A, \exists\left\{t_{k}\right\}_{k \in \mathbb{N}} \subset \mathbb{R}_{+}: z_{k} \rightarrow \bar{z}, t_{k} \downarrow 0,\left(z_{k}-\bar{z}\right) / t_{k} \rightarrow d\right\}
$$

as the (Bouligand) tangent cone to $A$ at $\bar{z}$. Based on that, let us introduce

$$
\begin{aligned}
& \widehat{\mathcal{N}}_{A}(\bar{z}):=\mathcal{T}_{A}(\bar{z})^{\circ}, \\
& \mathcal{N}_{A}(\bar{z}):=\left\{\xi \in \mathbb{R}^{n} \mid \begin{array}{c}
\exists\left\{z_{k}\right\}_{k \in \mathbb{N}} \subset A, \exists\left\{\xi_{k}\right\}_{k \in \mathbb{N}} \subset \mathbb{R}^{n}: \\
z_{k} \rightarrow \bar{z}, \xi_{k} \rightarrow \xi, \xi_{k} \in \widehat{\mathcal{N}}_{A}\left(z_{k}\right) \forall k \in \mathbb{N}
\end{array}\right\},
\end{aligned}
$$

the so-called regular (or Fréchet) and limiting (or Mordukhovich) normal cone to $A$ at $\bar{z}$, respectively. In case where $A$ is convex, these normal cones correspond to the normal cone in the sense of convex analysis, i.e., $\widehat{\mathcal{N}}_{A}(\bar{z})=\mathcal{N}_{A}(\bar{z})=(A-\bar{z})^{\circ}$.

For a locally Lipschitz continuous function $\varphi: \mathbb{R}^{n} \rightarrow \mathbb{R}$, we define its limiting subdifferential at some point $\bar{z} \in \mathbb{R}^{n}$ by means of

$$
\partial \varphi(\bar{z}):=\left\{\xi \in \mathbb{R}^{n} \mid(\xi,-1) \in \mathcal{N}_{\text {epi } \varphi}(\bar{z}, \varphi(\bar{z}))\right\}
$$

where epi $\varphi:=\left\{(z, s) \in \mathbb{R}^{n} \times \mathbb{R} \mid s \geq \varphi(z)\right\}$ denotes the epigraph of $\varphi$. In case where $\varphi$ is convex, the above arguments yield that $\partial \varphi(\bar{z})$ coincides with the subdifferential in the sense of convex analysis, i.e.,

$$
\partial \varphi(\bar{z})=\left\{\xi \in \mathbb{R}^{n} \mid \forall z \in \mathbb{R}^{n}: \varphi(z) \geq \varphi(\bar{z})+\xi^{\top}(z-\bar{z})\right\}
$$

holds in this case.

For a set-valued mapping $\Upsilon: \mathbb{R}^{n} \rightrightarrows \mathbb{R}^{m}$ with closed graph and some point $(\bar{z}, \bar{w}) \in$ gph $\Upsilon$, the set-valued mapping $D^{*} \Upsilon(\bar{z}, \bar{w}): \mathbb{R}^{m} \rightrightarrows \mathbb{R}^{n}$ given by

$$
\forall \eta \in \mathbb{R}^{m}: \quad D^{*} \Upsilon(\bar{z}, \bar{w})(\eta):=\left\{\xi \in \mathbb{R}^{n} \mid(\xi,-\eta) \in \mathcal{N}_{\mathrm{gph} \Upsilon}(\bar{z}, \bar{w})\right\}
$$


is referred to as the (limiting) coderivative of $\Upsilon$ at $(\bar{z}, \bar{w})$. For a single-valued mapping $v: \mathbb{R}^{n} \rightarrow \mathbb{R}^{m}$, we exploit the notation $D^{*} v(\bar{z}):=D^{*} v(\bar{z}, v(\bar{z}))$. Whenever $v$ is continuously differentiable at $\bar{z}$, then $D^{*} v(\bar{z})(\eta)=\nabla v(\bar{z})^{\top} \eta$ holds for all $\eta \in \mathbb{R}^{m}$ where $\nabla v(\bar{z})$ denotes the Jacobian of $v$ at $\bar{z}$.

Let us recall that $\Upsilon$ possesses the Aubin property at $(\bar{z}, \bar{w})$ if and only if the condition

$$
D^{*} \Upsilon(\bar{z}, \bar{w})(0)=\{0\}
$$

holds. Thus, $\Upsilon$ is metrically regular at $(\bar{z}, \bar{w})$ if and only if we have

$$
\operatorname{ker} D^{*} \Upsilon(\bar{z}, \bar{w})=\{0\} .
$$

Both criteria are referred to as Mordukhovich criterion in the literature, see [48, Theorem 3.3].

\subsection{M-STATIONARITY FOR OPTIMIZATION PROBLEM WITH GENERALIZED EQUATION CONSTRAINTS}

The following result can be found in [37, Theorem 4.1] and [33, Proposition 4.1]. It provides a calculus rule for the computation of the limiting normal cone to the pre-image associated with a set-valued mapping which is metrically subregular at a certain point of interest.

Proposition 3.1. Let $\Upsilon: \mathbb{R}^{n} \rightrightarrows \mathbb{R}^{m}$ be a set-valued mapping having locally closed graph around $(\bar{z}, \bar{w}) \in$ gph $\Upsilon$ and assume that $\Upsilon$ is metrically subregular at $(\bar{z}, \bar{w})$ with modulus $\bar{\kappa}$. Then the following estimate is valid for each $\kappa>\bar{\kappa}$ :

$$
\mathcal{N}_{\Upsilon^{-1}(\bar{w})}(\bar{z}) \subset\left\{\xi \in \mathbb{R}^{n} \mid \exists \eta \in \kappa\|\xi\| \mathbb{B}: \xi \in D^{*} \Upsilon(\bar{z}, \bar{w})(\eta)\right\} .
$$

This motivates the subsequently stated extension of the definition of M-stationarity to abstract optimization programs of the form

$$
\begin{aligned}
\varphi(z) & \rightarrow \min \\
0 & \in \Upsilon(z),
\end{aligned}
$$

where $\varphi: \mathbb{R}^{n} \rightarrow \mathbb{R}$ is a locally Lipschitz continuous function and $\Upsilon: \mathbb{R}^{n} \rightrightarrows \mathbb{R}^{m}$ is a set-valued mapping with closed graph, observing that $\Upsilon^{-1}(0)$ is the feasible set of $(G)$. A similar stationarity condition has been studied in the literature, see [57, Section 3].

Definition 3.2. We say that a feasible point $\bar{z} \in \Upsilon^{-1}(0)$ of the program $(\mathrm{G})$ is M-stationary provided there exists $\eta \in \mathbb{R}^{m}$ such that

$$
0 \in \partial \varphi(\bar{z})+D^{*} \Upsilon(\bar{z}, 0)(\eta) .
$$

In case where the constraint mapping $\Upsilon$ is given in the form of a so-called "feasibility" mapping, i.e., $\Upsilon(z):=\Phi(z)-\Omega$ for all $z \in \mathbb{R}^{n}$, where, for simplicity, $\Phi: \mathbb{R}^{n} \rightarrow \mathbb{R}^{m}$ is a continuously differentiable mapping and $\Omega \subset \mathbb{R}^{m}$ is a closed set, Proposition 3.1 yields the standard pre-image calculus rule

$$
\mathcal{N}_{\Phi^{-1}(\Omega)}(z) \subset \nabla \Phi(z)^{\top} \mathcal{N}_{\Omega}(\Phi(z))
$$

for each $z \in \mathbb{R}^{n}$ such that $\Upsilon$ is metrically subregular at $(z, 0) \in$ gph $\Upsilon$. This also justifies the Mstationarity terminology in Definition 3.2. Moreover, the additional bound $\|\eta\| \leq \kappa\|\xi\|$ in (3.1) implies that for every $\xi \in \mathcal{N}_{\Phi^{-1}(\Omega)}(z)$, there exists a multiplier $\eta \in \mathcal{N}_{\Omega}(\Phi(z)) \cap \kappa\|\xi\| \mathbb{B}$ with $\xi=\nabla \Phi(z)^{\top} \eta$. This observation, however, brings forth the following important property of the associated multiplier mapping which has been discussed in [6, Theorem 3.9] recently.

Proposition 3.3. Let $\Phi: \mathbb{R}^{n} \rightarrow \mathbb{R}^{m}$ be continuously differentiable and let $\Omega \subset \mathbb{R}^{m}$ be closed. We consider $\Upsilon: \mathbb{R}^{n} \rightrightarrows \mathbb{R}^{m}$ given by $\Upsilon(z):=\Phi(z)-\Omega$ for each $z \in \mathbb{R}^{n}$. Let $\left(\bar{z}, \bar{z}^{*}\right) \in$ gph $\mathcal{N}_{C}$ for $C:=\Phi^{-1}(\Omega)$ be fixed and assume that the feasibility mapping $\Upsilon$ is metrically subregular at $(\bar{z}, 0)$. Then the multiplier mapping $\Lambda: \mathbb{R}^{n} \times \mathbb{R}^{n} \rightrightarrows \mathbb{R}^{m}$, given by

$$
\forall\left(z, z^{*}\right) \in \mathbb{R}^{n} \times \mathbb{R}^{n}: \quad \Lambda\left(z, z^{*}\right):=\left\{\eta \in \mathcal{N}_{\Omega}(\Phi(z)) \mid \nabla \Phi(z)^{\top} \eta=z^{*}\right\},
$$

is inner semicompact at $\left(\bar{z}, \bar{z}^{*}\right)$ w.r.t. gph $\mathcal{N}_{C}$. Moreover, $\operatorname{gph} \mathcal{N}_{C}=\operatorname{dom} \Lambda$ holds provided $\Omega$ is convex. 
For comparison, it is well known that the stronger assumption of metric regularity yields local boundedness of the multiplier mapping $\Lambda$, and this has indeed been often used to guarantee its inner semicompactness, see Section 5.1 as well. While it may seem that local boundedness is not much stronger than inner semicompactness, when the underlying assumptions are compared, the gap is noteworthy. In case of standard nonlinear programs, metric regularity equals the prominent Mangasarian-Fromovitz constraint qualification (MFCQ), while in case of the more general class of geometric constraints as discussed in Proposition 3.3, metric regularity of the feasibility mapping $\Upsilon$ at $(\bar{z}, 0) \in \operatorname{gph} \Upsilon$ boils down to

$$
\nabla \Phi(\bar{z})^{\top} \eta=0, \eta \in \mathcal{N}_{\Omega}(\Phi(\bar{z})) \quad \Longrightarrow \quad \eta=0,
$$

which is called generalized Mangasarian-Fromovitz constraint qualification (GMFCQ) or no nonzero abnormal multiplier constraint qualification (NNAMCQ). The above propositions often enable us to relax the metric regularity requirement to the so-called metric subregularity constraint qualification (MSCQ) demanding $\Upsilon$ to be metrically subregular at $(\bar{z}, 0)$.

\section{$3 \cdot 3$ CALCULUS OF SET-VALUED MAPPINGS}

Throughout this section, we assume that all considered set-valued mappings possess closed graphs.

We first review the chain rule for the computation of coderivatives of compositions. Therefore, let us consider set-valued mappings $S_{1}: \mathbb{R}^{n} \rightrightarrows \mathbb{R}^{m}$ and $S_{2}: \mathbb{R}^{m} \rightrightarrows \mathbb{R}^{s}$ as well as their composition $S_{2} \circ S_{1}: \mathbb{R}^{n} \rightrightarrows \mathbb{R}^{s}$ given by

$$
\forall z \in \mathbb{R}^{n}: \quad\left(S_{2} \circ S_{1}\right)(z):=\bigcup_{y \in S_{1}(z)} S_{2}(y) .
$$

In order to estimate the coderivative of $S_{2} \circ S_{1}$, we introduce the standard "intermediate" mapping $\Xi: \mathbb{R}^{n} \times \mathbb{R}^{s} \rightrightarrows \mathbb{R}^{m}$ given by

$$
\forall z \in \mathbb{R}^{n}, \forall w \in \mathbb{R}^{s}: \quad \Xi(z, w):=S_{1}(z) \cap S_{2}^{-1}(w)=\left\{y \in S_{1}(z) \mid w \in S_{2}(y)\right\},
$$

together with the "feasibility" mapping $\Upsilon: \mathbb{R}^{n} \times \mathbb{R}^{s} \times \mathbb{R}^{m} \rightrightarrows \mathbb{R}^{m} \times \mathbb{R}^{s}$ in the following form:

$$
\forall z \in \mathbb{R}^{n}, \forall w \in \mathbb{R}^{s}, \forall y \in \mathbb{R}^{m}: \quad \Upsilon(z, w, y):=\left(\begin{array}{c}
S_{1}(z)-y \\
S_{2}(y)-w
\end{array}\right) .
$$

The following chain rule is taken from [9, Theorem 5.2].

Lemma 3.4. Fix $(\bar{z}, \bar{w}) \in \operatorname{gph}\left(S_{2} \circ S_{1}\right)$. Let $\Xi$ be inner semicompact w.r.t. $\operatorname{dom} \Xi$ at $(\bar{z}, \bar{w})$ and let $\Upsilon$ be metrically subregular at all points $((\bar{z}, \bar{w}, \bar{y}),(0,0))$ such that $\bar{y} \in \Xi(\bar{z}, \bar{w})$. Then we have

$$
\forall w^{*} \in \mathbb{R}^{s}: \quad D^{*}\left(S_{2} \circ S_{1}\right)(\bar{z}, \bar{w})\left(w^{*}\right) \subset \bigcup_{\bar{y} \in \Xi(\bar{z}, \bar{w})}\left(D^{*} S_{1}(\bar{z}, \bar{y}) \circ D^{*} S_{2}(\bar{y}, \bar{w})\right)\left(w^{*}\right) .
$$

In [9, Theorem 5.2], the subregularity assumption is replaced by the equivalent calmness assumption associated with the inverse mapping of $\Upsilon$ given by

$$
\Upsilon^{-1}(a, b)=\left\{(z, w, y) \mid y+a \in S_{1}(z), w+b \in S_{2}(y)\right\} .
$$

Furthermore, note that $\Upsilon$ is slightly different from the typical feasibility mapping used in the chain rule, see e.g. [53, proof of Theorem 10.37]. However, it is worth to mention that the respective metric subregularity assumptions, which need to be postulated on these maps in order to infer the chain rule, are actually equivalent due to [9, Proposition 2.6]. 
The proof of Lemma 3.4 is based on the essential relations

$$
\operatorname{gph}\left(S_{2} \circ S_{1}\right)=\operatorname{dom} \Xi, \quad \operatorname{gph} \Xi=\Upsilon^{-1}(0,0) .
$$

Roughly speaking, the inner semicompactness of $\Xi$ provides a connection between the graph of $S_{2} \circ S_{1}$ and the graph of $\Xi$ while the metric subregularity of $\Upsilon$ connects the graph of $\Xi$ with the graph of $\Upsilon$ via Proposition 3.1, see [9, Sections 3 and 5.3] for details. The combination of these two assumptions, hence, yields a way to estimate the coderivative of $S_{2} \circ S_{1}$ via the coderivative of $\Upsilon$, namely

$$
\begin{aligned}
& z^{*} \in D^{*}\left(S_{2} \circ S_{1}\right)(\bar{z}, \bar{w})\left(w^{*}\right) \\
& \quad \Longrightarrow \exists \bar{y} \in \Xi(\bar{z}, \bar{w}), \exists \xi_{1} \in \mathbb{R}^{m}, \exists \xi_{2} \in \mathbb{R}^{s}:\left(z^{*},-w^{*}, 0\right) \in D^{*} \Upsilon((\bar{z}, \bar{w}, \bar{y}),(0,0))\left(\xi_{1}, \xi_{2}\right) .
\end{aligned}
$$

Finally, the coderivative of $\Upsilon$ can be expressed via its components as

$$
D^{*} \Upsilon((\bar{z}, \bar{w}, \bar{y}),(0,0))\left(\xi_{1}, \xi_{2}\right)=D^{*} S_{1}(\bar{z}, \bar{y})\left(\xi_{1}\right) \times\{0\} \times D^{*} S_{2}(\bar{y}, \bar{w})\left(\xi_{2}\right)-\left(0, \xi_{2}, \xi_{1}\right) .
$$

This can be seen from Lemma 3.7 (b) (whose proof does not depend on the chain rule) where one can split $(z, w, y)$ either as $((z, w), y)$ or as $(z,(w, y))$. The above formulas now readily yield the desired chain rule via the coderivatives of $S_{1}$ and $S_{2}$.

Naturally, in specific situations, one may prefer to use different intermediate and feasibility mappings satisfying (3.3). The question then remains whether one can handle the coderivative of the chosen feasibility mapping to derive the chain rule without additional assumptions.

For set-valued mappings $\Gamma_{1}: \mathbb{R}^{n} \rightrightarrows \mathbb{R}^{m_{1}}$ and $\Gamma_{2}: \mathbb{R}^{n} \rightrightarrows \mathbb{R}^{m_{2}}$, we consider the product mapping $\Gamma: \mathbb{R}^{n} \rightrightarrows \mathbb{R}^{m_{1}} \times \mathbb{R}^{m_{2}}$ given by

$$
\forall z \in \mathbb{R}^{n}: \quad \Gamma(z):=\Gamma_{1}(z) \times \Gamma_{2}(z) .
$$

For later use, we need to compute or at least estimate the coderivative of this mapping. From [9, Section 5.4], we obtain the next result.

Lemma 3.5. Fix $\left(\bar{z},\left(\bar{w}_{1}, \bar{w}_{2}\right)\right) \in$ gph $\Gamma$. Assume that $\widetilde{\Upsilon}: \mathbb{R}^{n} \times \mathbb{R}^{m_{1}} \times \mathbb{R}^{m_{2}} \times \mathbb{R}^{n} \times \mathbb{R}^{n} \rightrightarrows \mathbb{R}^{n} \times \mathbb{R}^{n} \times \mathbb{R}^{m_{1}} \times \mathbb{R}^{m_{2}}$, given by

$$
\forall z, q_{1}, q_{2} \in \mathbb{R}^{n}, \forall w_{1} \in \mathbb{R}^{m_{1}}, \forall w_{2} \in \mathbb{R}^{m_{2}}: \quad \widetilde{\Upsilon}\left(z, w_{1}, w_{2}, q_{1}, q_{2}\right):=\left(\begin{array}{c}
z-q_{1} \\
z-q_{2} \\
\Gamma_{1}\left(q_{1}\right)-w_{1} \\
\Gamma_{2}\left(q_{2}\right)-w_{2}
\end{array}\right),
$$

is metrically subregular at $\left(\left(\bar{z}, \bar{w}_{1}, \bar{w}_{2}, \bar{z}, \bar{z}\right),(0,0,0,0)\right)$. Then we have

$$
D^{*} \Gamma\left(\bar{z},\left(\bar{w}_{1}, \bar{w}_{2}\right)\right)\left(\xi_{1}, \xi_{2}\right) \subset D^{*} \Gamma_{1}\left(\bar{z}, \bar{w}_{1}\right)\left(\xi_{1}\right)+D^{*} \Gamma_{2}\left(\bar{z}, \bar{w}_{2}\right)\left(\xi_{2}\right) .
$$

Moreover, $\widetilde{\Upsilon}$ is metrically subregular at $\left(\left(\bar{z}, \bar{w}_{1}, \bar{w}_{2}, \bar{z}, \bar{z}\right),(0,0,0,0)\right)$ whenever $\Gamma_{1}$ and $\Gamma_{2}$ are polyhedral or if the qualification condition

$$
D^{*} \Gamma_{1}\left(\bar{z}, \bar{w}_{1}\right)(0) \cap\left(-D^{*} \Gamma_{2}\left(\bar{z}, \bar{w}_{2}\right)(0)\right)=\{0\}
$$

holds, in which case $\widetilde{\Upsilon}$ is even metrically regular at the point of interest.

The qualification condition (3.5) is clearly satisfied if one of the mappings $\Gamma_{i}, i=1,2$, possesses the Aubin property at $\left(\bar{z}, \bar{w}_{i}\right)$. In the following lemmas, we review more results from [9, Section 5.4] concerning some exemplary settings where (3.5) holds naturally.

Lemma 3.6. Fix $\left(\bar{z},\left(\bar{w}_{1}, \bar{w}_{2}\right)\right) \in$ gph $\Gamma$ and assume that one of the following conditions holds. Then (3.5) is satisfied and, thus, the estimate (3.4) is valid. 
(a) There are a locally Lipschitz continuous function $\gamma: \mathbb{R}^{n} \rightarrow \mathbb{R}^{m_{2}}$ as well as a closed set $\Omega \subset \mathbb{R}^{m_{2}}$ such that $\Gamma_{2}$ is given by $\Gamma_{2}(z):=\gamma(z)-\Omega$ for all $z \in \mathbb{R}^{n}$ (an analogous statements holds if $\Gamma_{1}$ admits such a representation).

(b) The variables $z$ can be decomposed as $z=\left(z_{1}, z_{2}\right) \in \mathbb{R}^{n_{1}} \times \mathbb{R}^{n_{2}}$. Furthermore, there are set-valued mappings $\widetilde{\Gamma}_{1}: \mathbb{R}^{n_{1}} \rightrightarrows \mathbb{R}^{m_{1}}$ and $\widetilde{\Gamma}_{2}: \mathbb{R}^{n_{2}} \rightrightarrows \mathbb{R}^{m_{2}}$ as well as locally Lipschitz continuous functions $\gamma_{1}: \mathbb{R}^{n_{2}} \rightarrow \mathbb{R}^{m_{1}}$ and $\gamma_{2}: \mathbb{R}^{n_{1}} \rightarrow \mathbb{R}^{m_{2}}$ such that

$$
\forall z=\left(z_{1}, z_{2}\right) \in \mathbb{R}^{n_{1}} \times \mathbb{R}^{n_{2}}: \quad \Gamma_{1}(z):=\widetilde{\Gamma}_{1}\left(z_{1}\right)+\gamma_{1}\left(z_{2}\right), \quad \Gamma_{2}(z):=\widetilde{\Gamma}_{2}\left(z_{2}\right)+\gamma_{2}\left(z_{1}\right) .
$$

We note that, in principle, the setting (a) can be viewed as an extreme case of setting (b) with $n_{1}=n$ and $n_{2}=0$. Naturally, this does not fit formally, but one could regard a set as a set-valued mapping from a zero-dimensional space and propose a suitable relation for its coderivative via the normal cone to the set.

In the specific settings outlined above, we can, in fact, get equality in (3.4) instead of inclusion if we strengthen the Lipschitzness of the single-valued parts to continuous differentiability, see [9, Lemma 5.7].

Lemma 3.7. Fix $\left(\bar{z},\left(\bar{w}_{1}, \bar{w}_{2}\right)\right) \in$ gph $\Gamma$. Then the following statements hold.

(a) Consider the setting (a) from Lemma 3.6 and assume that the function $\gamma$ is continuously differentiable. Then we have

$$
D^{*} \Gamma\left(\bar{z},\left(\bar{w}_{1}, \bar{w}_{2}\right)\right)\left(\xi_{1}, \xi_{2}\right)= \begin{cases}D^{*} \Gamma_{1}\left(\bar{z}, \bar{w}_{1}\right)\left(\xi_{1}\right)+\nabla \gamma(\bar{z})^{\top} \xi_{2} & \text { if } \xi_{2} \in \mathcal{N}_{\Omega}\left(\gamma(\bar{z})-\bar{w}_{2}\right), \\ \varnothing & \text { otherwise }\end{cases}
$$

(b) Consider the setting (b) from Lemma 3.6 and assume that the functions $\gamma_{1}$ and $\gamma_{2}$ are continuously differentiable. Then we have

$$
D^{*} \Gamma\left(\bar{z},\left(\bar{w}_{1}, \bar{w}_{2}\right)\right)\left(\xi_{1}, \xi_{2}\right)=\left(\begin{array}{l}
D^{*} \widetilde{\Gamma}_{1}\left(\bar{z}_{1}, \bar{w}_{1}-\gamma_{1}\left(\bar{z}_{2}\right)\right)\left(\xi_{1}\right)+\nabla \gamma_{2}\left(\bar{z}_{1}\right)^{\top} \xi_{2} \\
D^{*} \widetilde{\Gamma}_{2}\left(\bar{z}_{2}, \bar{w}_{2}-\gamma_{2}\left(\bar{z}_{1}\right)\right)\left(\xi_{2}\right)+\nabla \gamma_{1}\left(\bar{z}_{2}\right)^{\top} \xi_{1}
\end{array}\right)
$$

Up to now, we discussed the auxiliary issue of how to guarantee metric subregularity of $\widetilde{\Upsilon}$, while the main target is to justify the upper estimate for the coderivative of $\Gamma$. Later, we will also employ metric subregularity of the product mapping $\Gamma$ itself, which is, however, often a more difficult issue. We conclude the preliminary part by a simple result regarding how to ensure metric subregularity of $\Gamma$ from metric subregularity of its factors $\Gamma_{1}$ and $\Gamma_{2}$. Let us mention that there exists a much deeper result by Klatte and Kummer, see [42, Theorem 3.6], on calmness of an intersection of mappings, which can be equivalently stated in terms of metric subregularity of a product of mappings, and which has several very interesting applications. In particular, since $\widetilde{\Upsilon}$ also has the structure of a product, it can be utilized even in situations when (3.5) fails to hold. Moreover, as demonstrated in [1], it applies quite nicely to the setting discussed in Section 2.1. Nevertheless, for the purposes of this paper, we will only need the following result.

Lemma 3.8. Fix $\left(\bar{z},\left(\bar{w}_{1}, \bar{w}_{2}\right)\right) \in \operatorname{gph} \Gamma$ and assume that $\Gamma_{i}$ is metrically subregular at $\left(\bar{z}, \bar{w}_{i}\right)$ for $i=1,2$ and, moreover, that the mapping $\Sigma: \mathbb{R}^{n} \rightrightarrows \mathbb{R}^{n} \times \mathbb{R}^{n}$ given by

$$
\forall z \in \mathbb{R}^{n}: \quad \Sigma(z):=\left(z-\Gamma_{1}^{-1}\left(\bar{w}_{1}\right)\right) \times\left(z-\Gamma_{2}^{-1}\left(\bar{w}_{2}\right)\right)
$$

is metrically subregular at $(\bar{z},(0,0))$. Then $\Gamma$ is metrically subregular at $\left(\bar{z},\left(\bar{w}_{1}, \bar{w}_{2}\right)\right)$. 
Proof. By assumption, we find neighborhoods $U, U_{1}, U_{2} \subset \mathbb{R}^{n}$ of $\bar{z}$ and reals $\kappa, \kappa_{1}, \kappa_{2}>0$ satisfying

$$
\forall z \in U: \quad \operatorname{dist}\left(z, \Sigma^{-1}(0,0)\right) \leq \kappa \operatorname{dist}((0,0), \Sigma(z))
$$

as well as

$$
\forall z \in U_{i}: \quad \operatorname{dist}\left(z, \Gamma_{i}^{-1}\left(\bar{w}_{i}\right)\right) \leq \kappa_{i} \operatorname{dist}\left(\bar{w}_{i}, \Gamma_{i}(z)\right)
$$

for $i=1,2$. Due to $\Gamma^{-1}\left(\bar{w}_{1}, \bar{w}_{2}\right)=\Sigma^{-1}(0,0)$, we obtain

$$
\begin{aligned}
\operatorname{dist}\left(z, \Gamma^{-1}\left(\bar{w}_{1}, \bar{w}_{2}\right)\right) & \leq \kappa\left(\operatorname{dist}\left(z, \Gamma_{1}^{-1}\left(\bar{w}_{1}\right)\right)+\operatorname{dist}\left(z, \Gamma_{2}^{-1}\left(\bar{w}_{2}\right)\right)\right) \\
& \leq \kappa \max \left\{\kappa_{1}, \kappa_{2}\right\} \operatorname{dist}\left(\left(\bar{w}_{1}, \bar{w}_{2}\right), \Gamma(z)\right)
\end{aligned}
$$

for each $z \in U \cap U_{1} \cap U_{2}$, and this shows the claim.

Since $\Sigma$ from (3.6) has a simpler structure than $\Gamma$, its coderivative can be readily computed by Lemma 3.7. Particularly, we note that metric regularity of $\Sigma$ at $(\bar{z},(0,0))$ reads

$$
\mathcal{N}_{\Gamma_{1}^{-1}\left(\bar{w}_{1}\right)}(\bar{z}) \cap\left(-\mathcal{N}_{\Gamma_{2}^{-1}\left(\bar{w}_{2}\right)}(\bar{z})\right)=\{0\},
$$

and, by metric subregularity of $\Gamma_{i}$ at $\left(\bar{z}, \bar{w}_{i}\right), i=1,2$, as well as the pre-image rule from Proposition 3.1, the condition

$$
\operatorname{rge} D^{*} \Gamma_{1}\left(\bar{z}, \bar{w}_{1}\right) \cap\left(-\operatorname{rge} D^{*} \Gamma_{2}\left(\bar{z}, \bar{w}_{2}\right)\right)=\{0\}
$$

is sufficient to guarantee its validity.

\section{ABSTRACT ANALYSIS WITH AND WITHOUT IMPLICIT VARIABLES}

In this section, we first address the relationship between the minimizers of $(\mathrm{P})$ and $(\mathrm{Q})$, respectively. Afterwards, we will deal with the derivation of necessary optimality conditions and constraint qualifications for $(\mathrm{P})$ with the aid of limiting variational analysis. In order to infer optimality conditions in terms of initial problem data (particularly, in terms of the generalized derivatives of $F$ and $G$ ), we use the chain and product rule of coderivative calculus from Section 3.3. As we will see, the associated assumptions for their respective application are valid in several practically relevant settings, which shows that the essential constraint qualification boils down to one subregularity assumption. We close the section with some brief remarks regarding sufficiency of the derived optimality conditions for convex problems of type (P).

\subsection{SOLUTION BEHAVIOR}

Here, we want to study the relationship between the local and global minimizers of $(\mathrm{P})$ and $(\mathrm{Q})$, respectively. To this end, the intermediate mapping $K: \mathbb{R}^{n} \rightrightarrows \mathbb{R}^{m}$ given by

$$
\forall z \in \mathbb{R}^{n}: \quad K(z):=\{\lambda \in F(z) \mid 0 \in G(z, \lambda)\}
$$

will be of essential importance. Note that we have $Z=M \cap \operatorname{dom} K$ by definition.

Lemma 4.1. If $F$ is a locally bounded set-valued mapping, then $H$ possesses a closed graph.

Proof. Let $\left\{\left(z_{k}, w_{k}\right)\right\}_{k \in \mathbb{N}} \subset$ gph $H$ be a sequence converging to some $(\bar{z}, \bar{w}) \in \mathbb{R}^{n} \times \mathbb{R}^{s}$. Then, by definition of $H$, for each $k \in \mathbb{N}$, we find $\lambda_{k} \in F\left(z_{k}\right)$ such that $w_{k} \in G\left(z_{k}, \lambda_{k}\right)$ holds. Since $F$ is locally bounded at $\bar{z},\left\{\lambda_{k}\right\}_{k \in \mathbb{N}}$ must be bounded. Thus, along a subsequence (without relabeling) it converges to some $\bar{\lambda} \in \mathbb{R}^{m}$. Recalling that $F$ and $G$ are set-valued mappings with a closed graph, $\bar{\lambda} \in F(\bar{z})$ and $\bar{w} \in G(\bar{z}, \bar{\lambda})$ follow, i.e., $\bar{w} \in H(\bar{z})$ is obtained. This shows the closedness of gph $H$. 
Exemplary, let us mention that in the setting of Section 2.1, the mapping $F$ is cone-valued and not likely to be locally bounded. However, one can check that the associated mapping $H$ still possesses a closed graph by closedness of the normal cone mapping associated with a convex set.

Subsequently, we discuss some properties of the mapping $K$.

Lemma 4.2. The following assertions hold.

(a) The mapping $K$ possesses a closed graph.

(b) Fix $\bar{z} \in \operatorname{dom} K$. If $F$ is locally bounded at $\bar{z}$, then $K$ is inner semicompact at $\bar{z}$ w.r.t. $\operatorname{dom} K$.

Proof. Let us start with the proof of assertion (a). Let $\left\{\left(z_{k}, \lambda_{k}\right)\right\}_{k \in \mathbb{N}} \subset$ gph $K$ be chosen such that $z_{k} \rightarrow \bar{z}$ and $\lambda_{k} \rightarrow \bar{\lambda}$ hold. By definition, we have $\lambda_{k} \in F\left(z_{k}\right)$ and $0 \in G\left(z_{k}, \lambda_{k}\right)$ for each $k \in \mathbb{N}$. The closedness of gph $F$ and gph $G$ yields $\bar{\lambda} \in F(\bar{z})$ and $0 \in G(\bar{z}, \bar{\lambda})$, respectively. Hence, we have $\bar{\lambda} \in K(\bar{z})$, i.e., gph $K$ is closed.

In order to show (b), let $\left\{z_{k}\right\}_{k \in \mathbb{N}} \subset \operatorname{dom} K$ be chosen such that $z_{k} \rightarrow \bar{z}$ holds. Then we find a sequence $\left\{\lambda_{k}\right\}_{k \in \mathbb{N}} \subset \mathbb{R}^{m}$ such that $\lambda_{k} \in K\left(z_{k}\right)$ holds for all $k \in \mathbb{N}$. Noting that $K$ is locally bounded at $\bar{z}$ since $F$ enjoys this property by assumption, $\left\{\lambda_{k}\right\}_{k \in \mathbb{N}}$ is bounded and possesses a convergent subsequence. Thus, $K$ is inner semicompact at $\bar{z}$ w.r.t. $\operatorname{dom} K$.

In the upcoming theorem, we take a look at the global minimizers of (P) and (Q).

Theorem 4.3.

(a) Let $\bar{z} \in \mathbb{R}^{n}$ be a global minimizer of $(\mathrm{P})$. Then, for each $\lambda \in K(\bar{z}),(\bar{z}, \lambda)$ is a global minimizer of (Q).

(b) Let $(\bar{z}, \bar{\lambda}) \in \mathbb{R}^{n} \times \mathbb{R}^{m}$ be a global minimizer of $(\mathrm{Q})$. Then $\bar{z}$ is a global minimizer of $(\mathrm{P})$.

Proof. Both statements of the theorem follow from the observation that for given $z \in \mathbb{R}^{n}$, we have the equivalences

$$
z \in Z \Longleftrightarrow z \in M \cap \operatorname{dom} K \Longleftrightarrow \exists \lambda \in \mathbb{R}^{m}:(z, \lambda) \in \tilde{Z},
$$

while the objective function of (Q) does not depend on the variable $\lambda$.

The above theorem shows that the relationship between $(\mathrm{P})$ and $(\mathrm{Q})$ is straight whenever global minimizers are under consideration. Thus, whenever these problems actually do not possess local minimizers which are not globally optimal, then $(\mathrm{P})$ and $(\mathrm{Q})$ are equivalent w.r.t. their minimizers in the sense of Theorem 4.3.

Corollary 4.4. Consider the situation where $F$ and $G$ are convex set-valued mappings. One can easily check that this implies convexity of $H$. Particularly, whenever $f$ is a convex function and $M$ is a convex set, then $(\mathrm{P})$ and $(\mathrm{Q})$ are both convex optimization problems (i.e., their objective function and feasible set are convex, respectively) whose minimizers correspond to each other in the sense of Theorem 4.3.

A quite popular and nearby situation which is addressed by Corollary 4.4 arises in the context of (conic) linear programming where slack variables are used to transfer a given problem instance into standard normal form. It is well known that the original problem and its surrogate are equivalent w.r.t. their minimizers.

Next, we investigate the relationship between local minimizers of $(\mathrm{P})$ and $(\mathrm{Q})$. As we will see, this issue is much more delicate in comparison with the situation where globally optimal solutions of these programs are under consideration.

Theorem 4.5.

(a) Let $\bar{z} \in \mathbb{R}^{n}$ be a local minimizer of $(\mathrm{P})$. Then, for each $\lambda \in K(\bar{z}),(\bar{z}, \lambda)$ is a local minimizer of (Q). 
(b) Let $(\bar{z}, \lambda) \in \mathbb{R}^{n} \times \mathbb{R}^{m}$ be a local minimizer of $(\mathrm{Q})$ for each $\lambda \in K(\bar{z})$. Furthermore, let $K$ be inner semicompact at $\bar{z}$ w.r.t. $\operatorname{dom} K$. Then $\bar{z}$ is a local minimizer of $(\mathrm{P})$.

Proof. For the proof of (a), suppose that, for some local minimizer $\bar{z} \in Z$ of (P), there is some $\bar{\lambda} \in K(\bar{z})$ such that $(\bar{z}, \bar{\lambda})$ is not a local minimizer of (Q). Then there is a sequence $\left\{\left(z_{k}, \lambda_{k}\right)\right\}_{k \in \mathbb{N}} \subset \tilde{Z}$ such that $z_{k} \rightarrow \bar{z}, \lambda_{k} \rightarrow \bar{\lambda}$, and $f\left(z_{k}\right)<f(\bar{z})$ holds for each $k \in \mathbb{N}$. Since we have $z_{k} \in M \cap \operatorname{dom} K$ by definition of $K, z_{k}$ is feasible to (P) for each $k \in \mathbb{N}$. Thus, $\bar{z}$ cannot be a local minimizer of (P) which is a contradiction. This shows (a).

For the proof of (b), let $(\bar{z}, \lambda) \in \mathbb{R}^{n} \times \mathbb{R}^{m}$ be a local minimizer of (Q) for each $\lambda \in K(\bar{z})$ and let $K$ be inner semicompact at $\bar{z}$ w.r.t. $\operatorname{dom} K$. From $\bar{z} \in M \cap \operatorname{dom} K$ we infer the feasibility of $\bar{z}$ to (P). Suppose now that $\bar{z}$ is not a local minimizer of (P). Then we find a sequence $\left\{z_{k}\right\}_{k \in \mathbb{N}} \subset Z$ such that $z_{k} \rightarrow \bar{z}$ while $f\left(z_{k}\right)<f(\bar{z})$ is satisfied for all $k \in \mathbb{N}$. From $z_{k} \in Z$ we deduce $z_{k} \in M \cap \operatorname{dom} K$ for each $k \in \mathbb{N}$. Due to the inner semicompactness of $K$ at $\bar{z}$ w.r.t. $\operatorname{dom} K$, there are a subsequence $\left\{z_{k_{l}}\right\}_{l \in \mathbb{N}}$ and a sequence $\left\{\lambda_{l}\right\}_{l \in \mathbb{N}} \subset \mathbb{R}^{m}$ converging to some $\bar{\lambda} \in \mathbb{R}^{m}$ such that $\lambda_{l} \in K\left(z_{k_{l}}\right)$ holds for all $l \in \mathbb{N}$. Since we have $z_{k_{l}} \rightarrow \bar{z}$, we can deduce $\bar{\lambda} \in K(\bar{z})$ from the closedness of gph $K$, see Lemma 4.2. Thus, the points from $\left\{\left(z_{k_{l}}, \lambda_{l}\right)\right\}_{l \in \mathbb{N}}$ as well as $(\bar{z}, \bar{\lambda})$ are feasible to (Q). Since we have $f\left(z_{k_{l}}\right)<f(\bar{z})$ for all $l \in \mathbb{N}$, $(\bar{z}, \bar{\lambda})$ cannot be a local minimizer of $(Q)$. This, however, contradicts our assumptions.

Naturally, when dealing with the reformulated problem (Q), one can hardly expect to find $\bar{z}$ such that $(\bar{z}, \lambda)$ is a local minimizer for all $\lambda \in K(\bar{z})$. Typically, one simply finds a point $(\bar{z}, \bar{\lambda})$ which is locally optimal (even this is often too optimistic and one only gets a stationary point instead). This underlines that the approach via problem $(Q)$ is indeed quite far from ideal. The proof of the previous theorem yields that the situation can be saved if the stronger inner semicontinuity of $K$ is assumed.

Corollary 4.6. Let $(\bar{z}, \bar{\lambda}) \in \mathbb{R}^{n} \times \mathbb{R}^{m}$ be a local minimizer of $(Q)$ and let $K$ be inner semicontinuous at $(\bar{z}, \bar{\lambda})$ w.r.t. $\operatorname{dom} K$. Then $\bar{z}$ is a local minimizer of $(\mathrm{P})$.

Due to Lemma 4.2, the inner semicompactness of $K$ can be guaranteed via local boundedness of $F$. In the context of bilevel programming, see Section 2.1, we already mentioned that the mapping $F$ does not enjoy this property. However, $K$ is the Lagrange multiplier mapping associated with the lower level problem in this context, and the latter is known to be locally bounded under validity of the Mangasarian-Fromovitz constraint qualification which, thus, implies its inner semicompactness as well. Hence, Theorem 4.5 recovers the popular result [20, Theorem 3.2]. However, exploiting Proposition 3.3, we are in position to weaken this constraint qualification such that the resulting multiplier mapping still possesses the necessary inner semicompactness, i.e., we will keep the essential result of Theorem 4.5 in this case, see Section 5.1 for details.

Remark 4.7. We point out that, in several exemplary cases, the inner semicompactness assumption will be automatically fulfilled (or will be a consequence of some initial assumption on the data, such as validity of a constraint qualification for the lower level program in bilevel programming). In fact, even a stronger form of inner semicompactness considered in Section 4.2 (i.e., inner semicompactness w.r.t. another intermediate mapping) will hold true, see Section 5.1 as well.

Below, we illustrate that none of the assumptions which are necessary in order to identify a local minimizer of $(\mathrm{P})$ via local minimizers of $(\mathrm{Q})$ can be dropped in Theorem 4.5.

Example 4.8.

(a) Let us consider the setting $n=m=s=1$ with $f:=\mathrm{id}$,

$$
\forall z, \lambda \in \mathbb{R}: \quad F(z):=\left\{\begin{array}{ll}
\{0\} & z>0, \\
\{0,1\} & z=0, \\
\{1\} & z<0,
\end{array} \quad G(z, \lambda):=[-z-\lambda, \infty),\right.
$$


as well as $M:=\mathbb{R}$. Obviously, we have $Z=[-1, \infty)$, i.e., $\bar{z}:=-1$ is the uniquely determined local and global minimizer of the associated problem $(\mathrm{P})$. It holds

$$
\forall z \in \mathbb{R}: \quad K(z)= \begin{cases}\{0\} & z>0, \\ \{0,1\} & z=0, \\ \{1\} & z \in[-1,0), \\ \varnothing & z<-1 .\end{cases}
$$

At $\tilde{z}:=0, K$ is inner semicompact w.r.t. $\operatorname{dom} K=Z$. It can be easily checked that $(\tilde{z}, 0)$ is a local minimizer of (Q) while $(\tilde{z}, 1)$ is not. Thus, assertion (b) of Theorem 4.5 does not generally hold whenever the local optimality for $(Q)$ cannot be guaranteed for all realizations of the implicit variables in question.

(b) For $n=m=s=1$, we set $f:=\mathrm{id}$,

$$
\forall z, \lambda \in \mathbb{R}: \quad F(z):=\left\{\begin{array}{ll}
\{0\} & z \geq 0, \\
\{-1 / z\} & z<0,
\end{array} \quad G(z, \lambda):=[-1,1+z],\right.
$$

as well as $M:=\mathbb{R}$. Again, we find $Z=[-1, \infty)$, and $\bar{z}:=-1$ is the uniquely determined local and global minimizer of the associated problem $(\mathrm{P})$. One easily computes

$$
\forall z \in \mathbb{R}: \quad K(z)= \begin{cases}\{0\} & z \geq 0, \\ \{-1 / z\} & z \in[-1,0), \\ \varnothing & z<-1 .\end{cases}
$$

Let us consider $\tilde{z}:=0$. At $\tilde{z}, K$ is not inner semicompact w.r.t. $\operatorname{dom} K$. On the other hand, $(\tilde{z}, 0)$ is a local minimizer of (Q) since for $z \uparrow 0$, we have $\lambda \rightarrow \infty$ for any $\lambda \in K(z)$. Consequently, we cannot abstain from postulating inner semicompactness of $K$ in order to guarantee validity of assertion (b) of Theorem 4.5.

The issues regarding the relationship between local minimizers of $(\mathrm{P})$ and $(\mathrm{Q})$ result from the fact that the variable $\lambda$ is equipped with a metric structure in $(Q)$. This is quite natural when treating $(Q)$ as an optimization problem and forms the base for the derivation of necessary optimality conditions, constraint qualifications, and solution algorithms. In the context of bilevel optimization, the authors of [58] used a different approach by disregarding the metric structure w.r.t. the lower level Lagrange multipliers in a reformulation of the bilevel programming problem which exploits the optimal value function as well as the Karush-Kuhn-Tucker-conditions of the lower level problem simultaneously, see [58, Proposition 3.1]. In the more general setting considered here, this amounts to saying that a feasible point $(\bar{z}, \bar{\lambda}) \in \mathbb{R}^{n} \times \mathbb{R}^{m}$ of $(\mathrm{Q})$ is a local minimizer whenever there is a neighborhood $U \subset \mathbb{R}^{n}$ of $\bar{z}$ such that

$$
\forall(z, \lambda) \in \tilde{Z} \cap\left(U \times \mathbb{R}^{m}\right): \quad f(\bar{z}) \leq f(z)
$$

holds. One can easily check that whenever $\bar{z} \in \mathbb{R}^{n}$ is a local minimizer of $(\mathrm{P})$, then, for each $\lambda \in K(\bar{z})$, $(\bar{z}, \lambda)$ is a local minimizer of (Q) in the sense of $(4 \cdot 1)$. Conversely, if $(\bar{z}, \bar{\lambda}) \in \mathbb{R}^{n} \times \mathbb{R}^{m}$ is a local minimizer of (Q) in the sense of (4.1), then $\bar{z}$ is a local minimizer of (P). At the first glance, this approach seems to solve all the issues observed in Theorem 4.5. However, it is completely unclear how local minimizers of (Q) in the sense of (4.1) can be characterized or numerically identified. In [58], the authors do not comment on this shortcoming at all. Thus, although faced with the disadvantages carved out in Theorem 4.5, we will stick to the classical notion of local optimality in (Q), i.e., we impose a metric structure on $\lambda$ there.

We conclude this section with a brief remark regarding sufficient optimality conditions. 
Remark 4.9. In optimization theory, sufficient optimality conditions generally imply local linear or quadratic growth, i.e., they impose local isolatedness of the characterized minimizer. Now, consider a local minimizer $\bar{z} \in Z$ of $(\mathrm{P})$. Even if it is a locally isolated minimizer of (P), this property fails to hold for the respective local minimizer $(\bar{z}, \bar{\lambda})$ for each $\bar{\lambda} \in K(\bar{z})$ as soon as $K(\bar{z})$ is a connected set which is not a singleton since the objective of (Q) does not depend on the variable $\lambda$. As a consequence, one cannot rely on classical first- or second-order sufficient optimality conditions in order to characterize the minimizers of $(Q)$ as soon as $K$ is multivalued. In the light of Theorem 4.5 , it is, thus, difficult to infer local optimality of a feasible point of $(\mathrm{P})$ via $(\mathrm{Q})$. In the context of bilevel and cardinality-constrained programming, this phenomenon has been mentioned recently in [47, Remark 4.21] and [16, Section 3.2], respectively.

\subsection{NECESSARY OPTIMALITY CONDITIONS}

Here, we are going to investigate three notions of necessary optimality conditions of M-stationaritytype for program (P). Two of them correspond to (standard) M-stationarity defined in Definition 3.2 applied either directly to $(\mathrm{P})$ or to its reformulation $(\mathrm{Q})$, while the third notion offers conditions in a completely explicit form. Without mentioning it again, we assume throughout the section that gph $H$ is (locally) closed. As we have seen in Lemma 4.1 this is inherent if $F$ is locally bounded, but it also holds in far more general but practically relevant situations. In particular, gph $H$ is locally closed around $(\bar{z}, 0) \in \operatorname{gph} H$ if the mapping $\widehat{K}$ from (4.3) below is inner semicompact at $(\bar{z}, 0)$ w.r.t. $\operatorname{dom} \widehat{K}=\operatorname{gph} H$. For later use, let us define set-valued mappings $\mathcal{H}: \mathbb{R}^{n} \times \mathbb{R}^{m} \rightrightarrows \mathbb{R}^{m} \times \mathbb{R}^{s}$ and $\mathfrak{H}: \mathbb{R}^{n} \times \mathbb{R}^{m} \rightrightarrows \mathbb{R}^{n+m} \times \mathbb{R}^{n+m+s}$ by means of

$$
\forall z \in \mathbb{R}^{n}, \forall \lambda \in \mathbb{R}^{m}: \quad \mathcal{H}(z, \lambda):=\left(\begin{array}{c}
F(z)-\lambda \\
G(z, \lambda)
\end{array}\right), \quad \mathfrak{H}(z, \lambda):=\left(\begin{array}{c}
(z, \lambda)-\operatorname{gph} F \\
(z, \lambda, 0)-\operatorname{gph} G
\end{array}\right) .
$$

In order to incorporate the constraints $z \in M$, we will exploit the mappings $H_{M}: \mathbb{R}^{n} \rightrightarrows \mathbb{R}^{s} \times \mathbb{R}^{n}$, $\mathcal{H}_{M}: \mathbb{R}^{n} \times \mathbb{R}^{m} \rightrightarrows \mathbb{R}^{m} \times \mathbb{R}^{s} \times \mathbb{R}^{n}$, as well as $\mathfrak{H}_{M}: \mathbb{R}^{n} \times \mathbb{R}^{m} \rightrightarrows \mathbb{R}^{n+m} \times \mathbb{R}^{n+m+s} \times \mathbb{R}^{n}$ obtained from $H, \mathcal{H}$, and $\mathfrak{H}$ by adding $(z-M)$ as a component, i.e., we exemplary have $H_{M}(z):=H(z) \times(z-M)$ for all $z \in \mathbb{R}^{n}$. Observe that we have $z \in Z$ if and only if $(0,0) \in H_{M}(z)$ and $(z, \lambda) \in \tilde{Z}$ if and only if $(0,0,0) \in \mathcal{H}_{M}(z, \lambda)$ if and only if $((0,0),(0,0,0), 0) \in \mathfrak{H}_{M}(z, \lambda)$.

Definition 4.10. Let $\bar{z} \in \mathbb{R}^{n}$ be a feasible point of (P). Then $\bar{z}$ is said to be

(i) implicitly M-stationary if there exists $v \in \mathbb{R}^{s}$ such that

$$
0 \in \partial f(\bar{z})+D^{*} H(\bar{z}, 0)(v)+\mathcal{N}_{M}(\bar{z})
$$

(ii) fuzzily M-stationary (or fuzzily M-stationary w.r.t. $\bar{\lambda}$ ) if there exist $\bar{\lambda} \in K(\bar{z}), \mu \in \mathbb{R}^{m}$, and $v \in \mathbb{R}^{s}$ such that

$$
(0,0) \in \partial f(\bar{z}) \times\{0\}+D^{*} \mathcal{H}((\bar{z}, \bar{\lambda}),(0,0))(\mu, v)+\mathcal{N}_{M}(\bar{z}) \times\{0\}
$$

holds, i.e., if $(\bar{z}, \bar{\lambda})$ is M-stationary for $(Q)$,

(iii) explicitly M-stationary (or explicitly M-stationary w.r.t. $\bar{\lambda}$ ) if there exist $\bar{\lambda} \in K(\bar{z}), \mu \in \mathbb{R}^{m}$, and $v \in \mathbb{R}^{s}$ such that

$$
0 \in \partial f(\bar{z})+D^{*} F(\bar{z}, \bar{\lambda})(\mu)+\left\{\xi \in \mathbb{R}^{n} \mid(\xi, \mu) \in D^{*} G((\bar{z}, \bar{\lambda}), 0)(v)\right\}+\mathcal{N}_{M}(\bar{z}) .
$$

Proposition 3.1 now immediately provides constraint qualifications for the above M-stationarity notions.

Proposition 4.11. Let $\bar{z} \in \mathbb{R}^{n}$ be a local minimizer of (P). Then the following assertions hold. 
(a) If $H_{M}$ is metrically subregular at $(\bar{z},(0,0))$, then $\bar{z}$ is implicitly M-stationary.

(b) If $\mathcal{H}_{M}$ is metrically subregular at $((\bar{z}, \bar{\lambda}),(0,0,0))$ for some $\bar{\lambda} \in K(\bar{z})$, then $\bar{z}$ is fuzzily M-stationary w.r.t. $\bar{\lambda}$.

(c) If $\mathfrak{H}_{M}$ is metrically subregular at $((\bar{z}, \bar{\lambda}),((0,0),(0,0,0), 0))$ for some $\bar{\lambda} \in K(\bar{z})$, then $\bar{z}$ is explicitly M-stationary w.r.t. $\bar{\lambda}$.

Proof. Since $\bar{z}$ is a local minimizer of (P), [48, Theorem 6.1] guarantees validity of

$$
0 \in \partial f(\bar{z})+\mathcal{N}_{H_{M}^{-1}(0,0)}(\bar{z}) .
$$

Noting that for each $\lambda \in K(\bar{z}),(\bar{z}, \lambda)$ is a local minimizer of (Q), see Theorem 4.5, [48, Theorem 6.1] furthermore implies

$$
\begin{aligned}
& (0,0) \in \partial f(\bar{z}) \times\{0\}+\mathcal{N}_{\mathcal{H}_{M}^{-1}(0,0,0)}(\bar{z}, \bar{\lambda}), \\
& (0,0) \in \partial f(\bar{z}) \times\{0\}+\mathcal{N}_{\mathfrak{W}_{M}^{-1}((0,0),(0,0,0), 0)}(\bar{z}, \bar{\lambda}) .
\end{aligned}
$$

For the proof of (a), we exploit the metric subregularity of $H_{M}$ at $(\bar{z},(0,0))$ and Proposition 3.1 in order to find $0 \in \partial f(\bar{z})+D^{*} H_{M}(\bar{z},(0,0))(v, \xi)$ for some $v \in \mathbb{R}^{s}$ and $\xi \in \mathbb{R}^{n}$. Now, we can exploit assertion (a) of Lemma 3.7 in order to find

$$
D^{*} H_{M}(\bar{z},(0,0))(v, \xi)= \begin{cases}D^{*} H(\bar{z}, 0)(v)+\xi & \xi \in \mathcal{N}_{M}(\bar{z}), \\ \varnothing & \text { otherwise }\end{cases}
$$

and this shows that $\bar{z}$ is implicitly M-stationary.

The proof for (b) works in analogous way exploiting (4.2a).

In order to verify statement (c), we first introduce a continuously differentiable single-valued mapping $\mathfrak{h}_{M}: \mathbb{R}^{n} \times \mathbb{R}^{m} \rightarrow \mathbb{R}^{n+m} \times \mathbb{R}^{n+m+s} \times \mathbb{R}^{n}$ and a set $\Omega \subset \mathbb{R}^{n+m} \times \mathbb{R}^{n+m+s} \times \mathbb{R}^{n}$ by means of

$$
\forall z \in \mathbb{R}^{n}, \forall \lambda \in \mathbb{R}^{m}: \quad \mathfrak{h}_{M}(z, \lambda):=((z, \lambda),(z, \lambda, 0), z), \quad \Omega:=\operatorname{gph} F \times \operatorname{gph} G \times M
$$

and observe that $\mathfrak{G}_{M}(z, \lambda)=\mathfrak{h}_{M}(z, \lambda)-\Omega$ holds for all $z \in \mathbb{R}^{n}$ and $\lambda \in \mathbb{R}^{m}$. Thus, the metric subregularity of the feasibility mapping $\mathfrak{S}_{M}$ at the reference point guarantees applicability of the pre-image rule, see Section 3.2. Together with the product rule for the limiting normal cone, see [53, Proposition 6.41], we obtain

$$
\begin{aligned}
& \mathcal{N}_{\mathfrak{W}_{M}^{-1}((0,0),(0,0,0), 0)}(\bar{z}, \bar{\lambda}) \\
& \subset \nabla \mathfrak{h}_{M}(\bar{z}, \bar{\lambda})^{\top} \mathcal{N}_{\Omega}\left(\mathfrak{h}_{M}(\bar{z}, \bar{\lambda})\right) \\
& =\nabla \mathfrak{h}_{M}(\bar{z}, \bar{\lambda})^{\top}\left(\mathcal{N}_{\operatorname{gph} F}(\bar{z}, \bar{\lambda}) \times \mathcal{N}_{\operatorname{gph} G}(\bar{z}, \bar{\lambda}, 0) \times \mathcal{N}_{M}(\bar{z})\right)
\end{aligned}
$$

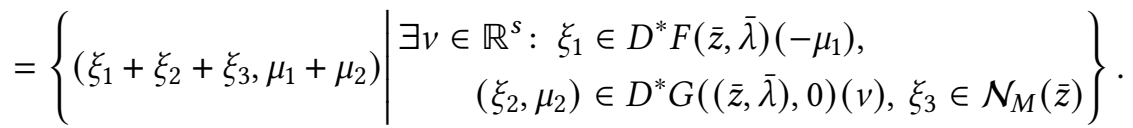

Now, the claim follows from (4.2b).

Remark 4.12. Taking into account Lemma 3.8 as well as the fact that the simple mapping $z \rightrightarrows z-M$ is trivially metrically subregular at all points of its graph, metric subregularity of $H_{M}$ at $(\bar{z},(0,0))$ can be ensured by metric subregularity of $H$ at $(\bar{z}, 0)$ and metric subregularity of $z \rightrightarrows\left(z-H^{-1}(0)\right) \times(z-M)$ at $(\bar{z},(0,0))$. Note that the latter implies applicability of the intersection rule for the set $Z=H_{M}^{-1}(0,0)=$ $H^{-1}(0) \cap M$, namely

$$
\mathcal{N}_{H_{M}^{-1}(0,0)}(\bar{z}) \subset \mathcal{N}_{H^{-1}(0)}(\bar{z})+\mathcal{N}_{M}(\bar{z})
$$

and, hence, combined with the metric subregularity of $H$, also provides a constraint qualification for implicit M-stationarity, see Proposition 3.1. 
Let us briefly comment on how to ensure the above metric subregularity assumptions via sufficient conditions in terms of coderivatives. Let $\bar{z} \in \mathbb{R}^{n}$ be a feasible point for $(\mathrm{P})$ and let $\bar{\lambda} \in K(\bar{z})$ be chosen arbitrarily.

(i) If, for all $v \in \mathbb{R}^{s}$ and $\xi \in \mathbb{R}^{n}$, the implication

$$
-\xi \in D^{*} H(\bar{z}, 0)(v) \cap\left(-\mathcal{N}_{M}(\bar{z})\right) \Longrightarrow \xi, v=0
$$

holds, then $H_{M}$ is metrically subregular at $(\bar{z},(0,0))$.

(ii) If, for all $\mu \in \mathbb{R}^{m}, v \in \mathbb{R}^{s}$, and $\xi \in \mathbb{R}^{n}$, the implication

$$
(-\xi, 0) \in D^{*} \mathcal{H}((\bar{z}, \bar{\lambda}),(0,0))(\mu, v), \xi \in \mathcal{N}_{M}(\bar{z}) \Longrightarrow \xi, \mu, v=0
$$

holds, then $\mathcal{H}_{M}$ is metrically subregular at $((\bar{z}, \bar{\lambda}),(0,0,0))$.

(iii) If, for all $\zeta \in \mathbb{R}^{n}, \mu \in \mathbb{R}^{m}, v \in \mathbb{R}^{s}$, and $\xi \in \mathbb{R}^{n}$, the implication

$$
\zeta \in D^{*} F(\bar{z}, \bar{\lambda})(\mu),(-\zeta-\xi, \mu) \in D^{*} G((\bar{z}, \bar{\lambda}), 0)(v), \xi \in \mathcal{N}_{M}(\bar{z}) \Longrightarrow \xi, \zeta, \mu, v=0
$$

holds, then $\mathfrak{H}_{M}$ is metrically subregular at $((\bar{z}, \bar{\lambda}),((0,0),(0,0,0), 0))$.

Observe that the above conditions correspond to the famous Mordukhovich criterion applied to the respective situation at hand. This can be seen from statement (a) of Lemma 3.7, the sum rule for coderivative calculus, see e.g. [48, Theorem 3.9], and the product rule for limiting normals, see [53, Proposition 6.41]. Thus, the above conditions already imply metric regularity of the mappings $H_{M}$, $\mathcal{H}_{M}$, and $\mathfrak{S}_{M}$ at the respective point of interest. Weaker sufficient conditions for the presence of metric subregularity in terms of limiting normal cones and coderivatives can exemplary be found in $[37,38,40,60]$. Even finer sufficient conditions can be obtained using the directional limiting approach, namely the first-order sufficient condition for metric subregularity from [30] or the directional pseudoand quasi-normality conditions from [3, 7]. For more details on the directional limiting approach in variational analysis, we exemplary refer to $[8,33]$ and the references therein.

Next, we are going to compare the three approaches on how to come up with necessary optimality conditions for $(\mathrm{P})$. First, we look at the qualification conditions and, afterwards, we deal with the stationarity conditions. For that purpose, let us define another intermediate mapping $\widehat{K}: \mathbb{R}^{n} \times \mathbb{R}^{s} \rightrightarrows \mathbb{R}^{m}$, closely related to $K$, given by

$$
\forall z \in \mathbb{R}^{n}, \forall w \in \mathbb{R}^{s}: \quad \widehat{K}(z, w):=\{\lambda \in F(z) \mid w \in G(z, \lambda)\} .
$$

Proposition 4.13. Let $\bar{z} \in \mathbb{R}^{n}$ be a feasible point of $(\mathrm{P})$. Consider the following two assumptions:

(a) $\mathcal{H}_{M}$ is metrically subregular at $((\bar{z}, \lambda),(0,0,0))$ for each $\lambda \in K(\bar{z})$ and $\widehat{K}$ is inner semicompact at $(\bar{z}, 0)$ w.r.t. $\operatorname{dom} \widehat{K}$,

(b) $\mathcal{H}_{M}$ is metrically subregular at $((\bar{z}, \bar{\lambda}),(0,0,0))$ for some $\bar{\lambda} \in K(\bar{z})$ and $\widehat{K}$ is inner semicontinuous at $((\bar{z}, 0), \bar{\lambda})$ w.r.t. $\operatorname{dom} \widehat{K}$.

Then each of the conditions (a) and (b) implies that $H_{M}$ is metrically subregular at $(\bar{z},(0,0))$.

Proof. Let us first show the statement under validity of (a). Suppose that $H_{M}$ is not metrically subregular at $(\bar{z},(0,0))$. Hence, for each $k \in \mathbb{N}$, we find $z_{k} \in \mathbb{R}^{n}$ and some $w_{k} \in H\left(z_{k}\right)$ satisfying

$$
\operatorname{dist}\left(z_{k}, Z\right)=\operatorname{dist}\left(z_{k}, H_{M}^{-1}(0,0)\right)>k \operatorname{dist}\left((0,0), H_{M}\left(z_{k}\right)\right)=k\left(\left\|w_{k}\right\|+\operatorname{dist}\left(z_{k}, M\right)\right)
$$


such that $z_{k} \rightarrow \bar{z}$. Particularly, $w_{k} \rightarrow 0$ follows. By definition of $H$ and $\widehat{K}$, we have $\left(z_{k}, w_{k}\right) \in \operatorname{dom} \widehat{K}$ for each $k \in \mathbb{N}$. The assumed inner semicompactness of $\widehat{K}$ at $(\bar{z}, 0)$ w.r.t. dom $\widehat{K}$ yields the existence of a sequence $\left\{\lambda_{k}\right\}_{k \in \mathbb{N}}$ and some $\lambda \in \mathbb{R}^{m}$ such that $\lambda_{k} \rightarrow \lambda$ and $\lambda_{k} \in \widehat{K}\left(z_{k}, w_{k}\right)$ hold along a subsequence (without relabeling). Recalling that $F$ and $G$ are mappings with closed graphs, the above convergences yield $\lambda \in F(\bar{z})$ and $0 \in G(\bar{z}, \lambda)$, i.e., $\lambda \in \widehat{K}(\bar{z}, 0)=K(\bar{z})$. Consequently, we obtain

$$
\begin{aligned}
\operatorname{dist} & \left(\left(z_{k}, \lambda_{k}\right), \mathcal{H}_{M}^{-1}(0,0,0)\right)=\operatorname{dist}\left(\left(z_{k}, \lambda_{k}\right), \tilde{Z}\right) \geq \operatorname{dist}\left(z_{k}, Z\right) \\
& >k\left(\left\|w_{k}\right\|+\operatorname{dist}\left(z_{k}, M\right)\right) \geq k\left(\operatorname{dist}\left(0, G\left(z_{k}, \lambda_{k}\right)\right)+\operatorname{dist}\left(z_{k}, M\right)\right) \\
& =k\left(\operatorname{dist}\left(0, F\left(z_{k}\right)-\lambda_{k}\right)+\operatorname{dist}\left(0, G\left(z_{k}, \lambda_{k}\right)\right)+\operatorname{dist}\left(z_{k}, M\right)\right) \\
& =k \operatorname{dist}\left((0,0,0), \mathcal{H}_{M}\left(z_{k}, \lambda_{k}\right)\right),
\end{aligned}
$$

showing that $\mathcal{H}_{M}$ is not metrically subregular at $((\bar{z}, \lambda),(0,0,0))$. This, however, contradicts the proposition's assumptions from (a).

The proof works similarly under validity of (b) observing that the sequence $\left\{\lambda_{k}\right\}_{k \in \mathbb{N}}$ can be chosen to be convergent to the fixed implicit variable $\bar{\lambda} \in \widehat{K}(\bar{z}, 0)$ by inner semicontinuity of $\widehat{K}$ w.r.t. $\operatorname{dom} \widehat{K}$ at $((\bar{z}, 0), \bar{\lambda})$.

Proposition 4.14. Let $\bar{z} \in \mathbb{R}^{n}$ be a feasible point of $(\mathrm{P})$ and fix $\bar{\lambda} \in K(\bar{z})$. Assume that $\mathfrak{H}_{M}$ is metrically subregular at $((\bar{z}, \bar{\lambda}),((0,0),(0,0,0), 0))$. Then $\mathcal{H}_{M}$ is metrically subregular at $((\bar{z}, \bar{\lambda}),(0,0,0))$.

Proof. The proof follows easily from $\mathcal{H}_{M}^{-1}(0,0,0)=\mathfrak{H}_{M}^{-1}((0,0),(0,0,0), 0)=\tilde{Z}$, together with the simple estimates

$$
\begin{array}{lrl}
\forall\left(z, \lambda^{\prime}\right) \in \operatorname{gph} F, \forall \lambda \in \mathbb{R}^{m}: & \operatorname{dist}((z, \lambda), \operatorname{gph} F) \leq\left\|\lambda-\lambda^{\prime}\right\|, \\
\forall((z, \lambda), w) \in \operatorname{gph} G: & \operatorname{dist}(((z, \lambda), 0), \operatorname{gph} G) \leq\|w\|
\end{array}
$$

which yield that $\operatorname{dist}\left(((0,0),(0,0,0), 0), \mathfrak{S}_{M}(z, \lambda)\right) \leq \operatorname{dist}\left((0,0,0), \mathcal{H}_{M}(z, \lambda)\right)$ holds.

Next, we investigate the relationship between the three stationarity notions from Definition 4.10 in more detail. Let us first address the relationship between fuzzy and explicit M-stationarity.

Remark 4.15. Lemmas 3.5 to 3.7 yield that, under suitable conditions, the coderivative of $\mathcal{H}$ at some point $((\bar{z}, \lambda),(0,0))$ of its graph can be estimated or computed via its components, i.e.,

$$
D^{*} \mathcal{H}((\bar{z}, \lambda),(0,0))(\mu, v) \subset D^{*} F(\bar{z}, \lambda)(\mu) \times\{-\mu\}+D^{*} G((\bar{z}, \lambda), 0)(v)
$$

for all $\mu \in \mathbb{R}^{m}$ and $v \in \mathbb{R}^{s}$. A sufficient condition for validity of $(\operatorname{Inc}(\lambda))$ is given by

$$
\xi \in D^{*} F(\bar{z}, \lambda)(0),(-\xi, 0) \in D^{*} G((\bar{z}, \lambda), 0)(0) \quad \Longrightarrow \quad \xi=0,
$$

see Lemma 3.5, and this is inherent if either $F$ possesses the Aubin property at $(\bar{z}, \lambda)$ or $G$ possesses the Aubin property at $((\bar{z}, \lambda), 0)$. Another situation where $(\operatorname{Inc}(\lambda))$ naturally holds is given in the case where $F$ and $G$ are polyhedral set-valued mappings. Note that $(\operatorname{Inc}(\lambda))$ does not hold for free in general due to fact that the variable $z$ enters both set-valued parts $F$ and $G$. Combining validity of $\operatorname{Inc}(\lambda))$ with simple computations provides conditions that guarantee that fuzzy M-stationarity implies or even coincides with explicit M-stationarity. We will show in Section 4.3 that the latter is automatically fulfilled for all of our example problems from Section 2, so we skip further details and consider these two notions to be basically identical.

Let us now tackle the more interesting question when an implicitly M-stationary point is fuzzily or explicitly M-stationary as well. The latter question can be easily answered by the chain rule since 
$H(z)=(G \circ \tilde{F})(z)$ holds for all $z \in \mathbb{R}^{n}$ where $\tilde{F}: \mathbb{R}^{n} \rightrightarrows \mathbb{R}^{n} \times \mathbb{R}^{m}$ is the set-valued mapping given by $\tilde{F}(z):=\{z\} \times F(z)$ for all $z \in \mathbb{R}^{n}$. Taking into account that

$$
D^{*} \tilde{F}(z,(z, \lambda))(\xi, \mu)=\xi+D^{*} F(z, \lambda)(\mu)
$$

holds by Lemma 3.7 (a), Lemma 3.4 immediately yields the following result.

Proposition 4.16. Let $\bar{z} \in \mathbb{R}^{n}$ be an implicitly $M$-stationary point for $(\mathrm{P})$. Then $\bar{z}$ is explicitly M-stationary provided the mapping

$$
(z, w) \rightrightarrows \tilde{F}(z) \cap G^{-1}(w)=\{(z, \lambda) \mid \lambda \in F(z), w \in G(z, \lambda)\}
$$

is inner semicompact at $(\bar{z}, 0)$ w.r.t. its domain while the mapping

$$
(z, w, q, \lambda) \rightrightarrows(z-q, F(z)-\lambda, G(q, \lambda)-w)
$$

is metrically subregular at $((\bar{z}, 0, \bar{z}, \lambda),(0,0,0))$ for each $\lambda \in K(\bar{z})$.

On the other hand, using the intermediate mapping $\widehat{K}$ from (4.3), one obtains

$$
\operatorname{gph} H=\operatorname{dom} \widehat{K}, \quad \operatorname{gph} \widehat{K}=\widehat{\mathcal{H}}^{-1}(0,0)
$$

for the feasibility mapping $\widehat{\mathcal{H}}: \mathbb{R}^{n} \times \mathbb{R}^{s} \times \mathbb{R}^{m} \rightrightarrows \mathbb{R}^{m} \times \mathbb{R}^{s}$ given by

$$
\forall z \in \mathbb{R}^{n}, \forall w \in \mathbb{R}^{s}, \forall \lambda \in \mathbb{R}^{m}: \quad \widehat{\mathcal{H}}(z, w, \lambda):=\left(\begin{array}{c}
F(z)-\lambda \\
G(z, \lambda)-w
\end{array}\right)=\mathcal{H}(z, \lambda)-\left(\begin{array}{l}
0 \\
w
\end{array}\right) .
$$

Keeping Lemma 3.4 and the subsequently stated remarks in mind, the coderivative of $H$ can be estimated via the coderivative of $\widehat{\mathcal{H}}$ under suitable assumptions. More precisely, for $(a, b)$ satisfying $a \in D^{*} H(\bar{z}, 0)(b)$, the above approach yields the existence of $\bar{\lambda} \in K(\bar{z})$ together with $\mu \in \mathbb{R}^{m}$ and $v \in \mathbb{R}^{s}$ such that

$$
(a,-b, 0) \in D^{*} \widehat{\mathcal{H}}((\bar{z}, 0, \bar{\lambda}),(0,0))(\mu, v) .
$$

Consequently, the decoupled sum rule from [9, Section 5.4] implies that the relations $v=b$ and $(a, 0) \in D^{*} \mathcal{H}((\bar{z}, \bar{\lambda}),(0,0))(\mu, v)$ need to hold, and for the particular choice $a \in-\partial f(\bar{z})-\mathcal{N}_{M}(\bar{z})$, we end up with fuzzy M-stationarity.

We summarize these observations in the subsequent proposition.

Proposition 4.17. Let $\bar{z} \in \mathbb{R}^{n}$ be an implicitly M-stationary point of $(\mathrm{P})$. Assume that $\widehat{K}$ is inner semicompact at $(\bar{z}, 0)$ w.r.t. $\operatorname{dom} \widehat{K}$, and let $\widehat{\mathcal{H}}$ be metrically subregular at $((\bar{z}, 0, \lambda),(0,0))$ for each $\lambda \in K(\bar{z})$. Then $\bar{z}$ is fuzzily M-stationary. Moreover, $\bar{z}$ is also explicitly M-stationary if $(\operatorname{Inc}(\lambda))$ holds for each $\lambda \in K(\bar{z})$.

The metric subregularity assumption in Proposition 4.17 can again be replaced by the stronger Mordukhovich criterion.

Corollary 4.18. Let $\bar{z} \in \mathbb{R}^{n}$ be an implicitly M-stationary point for (P). Let $\widehat{K}$ be inner semicompact at $(\bar{z}, 0)$ w.r.t. $\operatorname{dom} \widehat{K}$.

(a) Assume that, for each $\lambda \in K(\bar{z})$, the constraint qualification

$$
(0,0) \in D^{*} \mathcal{H}((\bar{z}, \lambda),(0,0))(\mu, 0) \quad \Longrightarrow \quad \mu=0
$$

holds, which is inherent whenever $\mathcal{H}$ is metrically regular at $((\bar{z}, \lambda),(0,0))$. Then $\bar{z}$ is fuzzily $M$-stationary. If, additionally, $(\operatorname{Inc}(\lambda))$ holds for each $\lambda \in K(\bar{z})$, then $\bar{z}$ is explicitly M-stationary. 
(b) Assume that $(\operatorname{Inc}(\lambda))$ holds for each $\lambda \in K(\bar{z})$ and let

$$
\xi \in D^{*} F(\bar{z}, \lambda)(\mu),(-\xi, \mu) \in D^{*} G((\bar{z}, \lambda), 0)(0) \quad \Longrightarrow \quad \mu=0
$$

be valid for each $\lambda \in K(\bar{z})$ which is inherent whenever $G$ posseses the Aubin property at $((\bar{z}, \lambda), 0)$ for each $\lambda \in K(\bar{z})$. Then $\bar{z}$ is explicitly M-stationary.

(c) Assume that

$$
\xi \in D^{*} F(\bar{z}, \lambda)(\mu),(-\xi, \mu) \in D^{*} G((\bar{z}, \lambda), 0)(0) \quad \Longrightarrow \quad \xi, \mu=0
$$

is valid for each $\lambda \in K(\bar{z})$ which is inherent whenever $G$ posseses the Aubin property at $((\bar{z}, \lambda), 0)$ for each $\lambda \in K(\bar{z})$. Then $\bar{z}$ is explicitly M-stationary.

Proof. Let us start with the proof of statement (a). The relation between the coderivatives of $\mathcal{H}$ and $\widehat{\mathcal{H}}$, which has been discussed above Proposition 4.17 , yields

$$
\operatorname{ker} D^{*} \widehat{\mathcal{H}}((\bar{z}, 0, \lambda),(0,0))=\left\{(\mu, 0) \mid(\mu, 0) \in \operatorname{ker} D^{*} \mathcal{H}((\bar{z}, \lambda),(0,0))\right\}
$$

for each $\lambda \in K(\bar{z})$. Thus, the constraint qualification (4.7) implies metric regularity of $\widehat{\mathcal{H}}$ at the point $((\bar{z}, 0, \lambda),(0,0))$ for each $\lambda \in K(\bar{z})$ by means of the Mordukhovich criterion. Thus, the assertion follows from Proposition 4.17. For the proof of (b), we observe that due to validity of $(\operatorname{Inc}(\lambda))$ for each $\lambda \in K(\bar{z})$, (4.8) implies (4.7) for each $\lambda \in K(\bar{z})$, i.e., the assertion follows from statement (a). Finally, (c) follows from (b) observing that (4.9) implies validity of (4.8) as well as $(\operatorname{Inc}(\lambda))$ by means of Remark 4.15.

Let us mention that, in general, explicitly M-stationary points do not need to be implicitly Mstationary, see e.g. Section 5.3 and [44, Section 4] where this issue is visualized in the context of cardinality- and or-constrained programming, respectively.

As a consequence of Propositions 4.11, 4.16 and 4.17, we obtain the following constraint qualifications which guarantee that a given local minimizer of $(\mathrm{P})$ is explicitly M-stationary.

Theorem 4.19. Let $\bar{z} \in \mathbb{R}^{n}$ be a local minimizer of $(\mathrm{P})$ and consider the following assumptions:

(a) $H_{M}$ is metrically subregular at $(\bar{z},(0,0)), \widehat{K}$ is inner semicompact at $(\bar{z}, 0)$ w.r.t. $\operatorname{dom} \widehat{K}$, and for each $\lambda \in K(\bar{z}), \widehat{\mathcal{H}}$ is metrically subregular at $((\bar{z}, 0, \lambda),(0,0))$ while the inclusion $(\operatorname{Inc}(\lambda))$ holds,

(b) $H_{M}$ is metrically subregular at $(\bar{z},(0,0))$, the mapping defined in $(4.4)$ is inner semicompact at $(\bar{z}, 0)$ w.r.t. its domain, and for each $\lambda \in K(\bar{z})$, the mapping defined in (4.5) is metrically subregular at $((\bar{z}, 0, \bar{z}, \lambda),(0,0,0))$,

(c) $\mathcal{H}_{M}$ is metrically subregular at $((\bar{z}, \bar{\lambda}),(0,0,0))$ for some $\bar{\lambda} \in K(\bar{z})$ and the inclusion $(\operatorname{Inc}(\bar{\lambda}))$ holds,

(d) $\mathfrak{H}_{M}$ is metrically subregular at $((\bar{z}, \bar{\lambda}),((0,0),(0,0,0), 0))$ for some $\bar{\lambda} \in K(\bar{z})$.

Then each of (a), (b), (c), and (d) implies that $\bar{z}$ is explicitly M-stationary.

Once more, let us emphasize that validity of the inclusion $(\operatorname{Inc}(\lambda))$ can be guaranteed under not too restrictive conditions which can be found in Lemmas 3.6 and 3.7, see Remark 4.15 as well.

In Section 4.3, we will see that the most essential ingredients from Theorem 4.19 are the metric subregularity assumptions on $H_{M}, \mathcal{H}_{M}$, and $\mathfrak{G}_{M}$, respectively, while the other requirements can often be guaranteed by simpler structure of the problem or by suitable qualification conditions.

A general purpose of necessary optimality conditions is to shrink the feasible set down to a (hopefully) small number of points which are potential candidates for local minimizers. Since we are interested in finding local minimizers $\bar{z} \in \mathbb{R}^{n}$ of $(\mathrm{P})$ even if (Q) is under consideration, Theorem 4.5 underlines that validity of explicit M-stationarity w.r.t. each $\bar{\lambda} \in K(\bar{z})$ is desirable for that purpose since this 
corresponds to standard M-stationarity for $(Q)$ at its local minimizers $(\bar{z}, \bar{\lambda})$ under mild assumptions, see Remark 4.15 again. In order to infer this directly from $(Q)$, one has to impose metric subregularity of $\mathfrak{H}_{M}$ at all points $((\bar{z}, \bar{\lambda}),((0,0),(0,0,0), 0))$ such that $\bar{\lambda} \in K(\bar{z})$ holds. Postulating that the intermediate mapping $\widehat{K}$ from (4.3) is inner semicompact w.r.t. dom $\widehat{K}$ at $(\bar{z}, 0)$ (which is inherent in several underlying applications), Propositions 4.13 and 4.14 guarantee that $H_{M}$ is metrically subregular at $(\bar{z},(0,0))$. Due to Proposition 4.11, this is already enough to ensure that the local minimizer $\bar{z}$ of $(\mathrm{P})$ is implicitly $\mathrm{M}$-stationary. In this regard, the constraint qualifications one has to impose on $(\mathrm{Q})$ in order to find potential candidates for local minimizers of $(\mathrm{P})$ via the associated $\mathrm{M}$-stationarity conditions are in several situations not weaker than the constraint qualifications one has to impose directly on $(\mathrm{P})$ in order to infer implicit M-stationarity as a necessary optimality condition. In the context of bilevel optimization, the counterexamples in [1, Section 3.1] depict that the converse statement of Proposition 4.13 does not hold in general. This also shows that, in some situations, the conditions in statement (a) of Theorem 4.19 might be weaker than the condition from statement (d) (but for all $\bar{\lambda} \in K(\bar{z})$ ) in this regard. Let us sum up some important points:

(i) it may happen that $\bar{z}$ is implicitly M-stationary while it is not explicitly M-stationary w.r.t. all elements of $K(\bar{z})$ (in case where the chain rule is applicable in order to compute the coderivative of $H$, one can only ensure explicit M-stationarity w.r.t. those implicit variables which are active in the union appearing in the chain rule), however, under mild assumptions, explicit M-stationarity for some instance of the implicit variable can be derived,

(ii) if $\bar{z}$ is a local minimizer of (P), then due to Theorem 4.5, explicit M-stationarity w.r.t. all elements of $K(\bar{z})$ is a reasonable necessary optimality condition for $(\mathrm{P})$ (under suitable constraint qualifications), and

(iii) the constraint qualifications needed to infer implicit M-stationarity of a local minimizer $\bar{z}$ of (P) ( $H_{M}$ metrically subregular at some reference point) and to obtain explicit M-stationarity from that, see Propositions 4.16 and 4.17 as well as Corollary 4.18, might still be weaker than the constraint qualifications needed to infer explicit M-stationarity w.r.t. all $\bar{\lambda} \in K(\bar{z})$ directly from (Q).

These observations underline that although the use of implicit variables as explicit ones might be beneficial for computational purposes, this transformation is disadvantageous since it comes for the price of additional artificial local minimizers and potentially stronger constraint qualifications. Instead of treating implicit variables as explicit ones, one should keep them implicit while exploiting the inherent underlying problem structure as long as possible in order to infer reasonably weak constraint qualifications ensuring validity of useful necessary optimality conditions at local minimizers.

Let us mention some positive features of implicit variables regarding optimality conditions. Clearly, whenever we are given some $\tilde{z} \in \mathbb{R}^{n}$ feasible to (P) and some $\tilde{\lambda} \in K(\tilde{z})$ such that $\mathfrak{H}_{M}$ is metrically subregular at $((\tilde{z}, \tilde{\lambda}),((0,0),(0,0,0), 0))$ while $\tilde{z}$ is not explicitly M-stationary w.r.t. $\tilde{\lambda}$, then $\tilde{z}$ cannot be a local minimizer of $(\mathrm{P})$, see Proposition 4.11. This means that implicit variables can be used to infer suboptimality conditions for (P). Secondly, let us mention that it might happen that a practically useful representation of the coderivative of $H$ is not available for some underlying applications while the computation of the coderivative of $F$ and $G$ might be possible in terms of initial problem data, see e.g. Section 5.2. Then the explicit M-stationarity conditions of (P) might be applicable while the implicit counterpart is of limited practical use. Of course, under rather mild assumptions one can estimate the coderivative of $H$ via the coderivatives of $F$ and $G$, but this is not completely for free in general.

\subsection{NECESSARY OPTIMALITY CONDITIONS UNDER ADDITIONAL STRUCTURAL ASSUMPTIONS}

In this section, we show how the situation from Section 4.2 simplifies in specific settings that cover our example problems from Section 2. To this end, we impose that the following assumption on the 
problem data holds throughout the section.

Assumption 4.20. The set gph $H$ is closed, and the problem data satisfies one of the following assumptions on the set-valued mapping $G$ :

(i) $G(z, \lambda):=g(z, \lambda)-\Theta$ holds for all $z \in \mathbb{R}^{n}$ and $\lambda \in \mathbb{R}^{m}$ where $g: \mathbb{R}^{n} \times \mathbb{R}^{m} \rightarrow \mathbb{R}^{s}$ is a continuously differentiable function and $\Theta \subset \mathbb{R}^{s}$ is a closed set,

(ii) $G(z, \lambda):=\widetilde{G}(\lambda)+\tilde{g}(z)$ holds for all $z \in \mathbb{R}^{n}$ and $\lambda \in \mathbb{R}^{m}$ where $\widetilde{G}: \mathbb{R}^{m} \rightrightarrows \mathbb{R}^{s}$ is a set-valued mapping with a closed graph and $\tilde{g}: \mathbb{R}^{n} \rightarrow \mathbb{R}^{s}$ is a continuously differentiable function.

Let us point out that (i) covers the setting of bilevel programming from (2.1) as well as the setting of cardinality-constrained optimization from (2.5), while (ii) covers the setting of evaluated multiobjective programming from (2.2).

Statements (a) and (b) of Lemma 3.7 yield that the inclusion $(\operatorname{Inc}(\lambda))$ holds with equality under any of the assumptions (i) or (ii). Thus, due to Remark 4.15, fuzzy M-stationarity in fact coincides with explicit M-stationarity and so we can work only with the latter. Particularly, we will only consider the implicit and explicit $\mathrm{M}$-stationarity conditions with the respective constraint qualification being metric subregularity of $H_{M}$ and $\mathcal{H}_{M}$.

For the remaining part, let us add the inner semicompactness of $\widehat{K}$ w.r.t. dom $\widehat{K}$ at $(\bar{z}, 0)$ for some feasible point $\bar{z} \in \mathbb{R}^{n}$ of $(\mathrm{P})$ to the standing assumption. Then the comparison of the metric subregularity conditions reduces to simply saying that $H_{M}$ is metrically subregular at $(\bar{z},(0,0))$ provided $\mathcal{H}_{M}$ is metrically subregular at $((\bar{z}, \lambda),(0,0,0))$ for all $\lambda \in K(\bar{z})$, see Proposition 4.13.

Finally, we discuss when implicit M-stationarity implies explicit M-stationarity. Again, the statements (a) and (b) of Lemma 3.7 show that there is now no problem with the computation of the coderivative of the mapping $\mathcal{H}$ and the same applies to the mapping $\widehat{\mathcal{H}}$ from (4.6) due to the arguments above Proposition 4.17. Particularly, we infer that $\widehat{\mathcal{H}}$ is metrically regular at $((\bar{z}, 0, \lambda),(0,0))$ for $\lambda \in K(\bar{z})$ if and only if the constraint qualification (4.8) is valid. One can check that the latter is inherently satisfied in the setting (i) since $G$ possesses the Aubin property in this case. If (ii) holds, (4.8) boils down to

$$
\operatorname{ker} D^{*} F(\bar{z}, \lambda) \cap D^{*} \widetilde{G}(\lambda,-\tilde{g}(\bar{z}))(0)=\{0\} .
$$

We sum up all these arguments in the following proposition.

Proposition 4.21. Let $\bar{z} \in \mathbb{R}^{n}$ be implicitly $M$-stationary for problem $(\mathrm{P})$ and let $\widehat{K}$ be inner semicompact w.r.t. $\operatorname{dom} \widehat{K}$ at $(\bar{z}, 0)$. Then the following assertions hold.

(a) Let $G$ be given as stated in (i). Then $\bar{z}$ is explicitly M-stationary, i.e., there exist $\bar{\lambda} \in K(\bar{z})$ and $v \in \mathcal{N}_{\Theta}(g(\bar{z}, \bar{\lambda}))$ such that

$$
0 \in \partial f(\bar{z})+D^{*} F(\bar{z}, \bar{\lambda})\left(\nabla_{\lambda} g(\bar{z}, \bar{\lambda})^{\top} v\right)+\nabla_{z} g(\bar{z}, \bar{\lambda})^{\top} v+\mathcal{N}_{M}(\bar{z}) .
$$

(b) Let $G$ be given as stated in (ii) and let $\widehat{\mathcal{H}}$ be metrically subregular at $((\bar{z}, 0, \lambda),(0,0))$ for each $\lambda \in K(\bar{z})$. Then $\bar{z}$ is explicitly $M$-stationary, i.e., there exist $\bar{\lambda} \in K(\bar{z})$ and $v \in \mathbb{R}^{s}$ such that

$$
0 \in \partial f(\bar{z})+\left(D^{*} F(\bar{z}, \bar{\lambda}) \circ D^{*} \widetilde{G}(\bar{\lambda},-\tilde{g}(\bar{z}))\right)(v)+\nabla \tilde{g}(\bar{z})^{\top} v+\mathcal{N}_{M}(\bar{z}) .
$$

The above proposition shows that in the setting (i) from Assumption 4.20, one only has to impose metric subregularity of $H_{M}$ at some reference point $(\bar{z},(0,0))$, where $\bar{z}$ is a local minimizer of $(\mathrm{P})$, and some inner semicompactness of $\widehat{K}$ in order to come up with fully explicit optimality conditions in terms of initial problem data. In the setting (ii), an additional metric subregularity requirement on $\widehat{\mathcal{H}}$ is necessary for that purpose. As we will see, inner semicompactness of $\widehat{K}$ is inherent under reasonable assumptions in the context of the problem settings from Section 2. Thus, one might be tempted to say that in any of the settings from Assumption 4.20, an implicitly M-stationary point of $(\mathrm{P})$ is likely to be explicitly M-stationary w.r.t. at least one choice of the implicit variable. 


\subsection{CONVEXITY AND SUFFICIENT OPTIMALITY CONDITIONS}

We want to close our theoretical analysis of the abstract model (P) with a brief look at sufficient optimality conditions in the presence of convexity. More precisely, we investigate the problem of interest under the subsequently stated standing assumption.

Assumption 4.22. Let $f$ be a convex function, let $F$ and $G$ be convex set-valued mappings, and let $M$ be a convex set.

As we already mentioned in Corollary 4.4, Assumption 4.22 ensures that $H$ is convex as well. Observing that the graphs of $F, G$, and $H$ are convex, their coderivatives are given via the normal cone in the sense of convex analysis. That is why we obtain the following result.

Theorem 4.23. Let $\bar{z} \in \mathbb{R}^{n}$ be an implicitly, fuzzily, or explicitly M-stationary point of (P). Then $\bar{z}$ is a minimizer of $(\mathrm{P})$.

Proof. We only show the statement for explicitly M-stationary points. The remaining assertions can be derived similarly.

Let $\bar{z}$ be explicitly M-stationary. Then we find $\bar{\lambda} \in K(\bar{z}), \mu \in \mathbb{R}^{m}$, and $v \in \mathbb{R}^{s}$ as well as $\xi_{1} \in \partial f(\bar{z})$, $\xi_{2} \in D^{*} F(\bar{z}, \bar{\lambda})(\mu),\left(\xi_{3}, \mu\right) \in D^{*} G((\bar{z}, \bar{\lambda}), 0)(v)$, and $\xi_{4} \in \mathcal{N}_{M}(\bar{z})$ with $0=\xi_{1}+\xi_{2}+\xi_{3}+\xi_{4}$. For each $(z, \lambda) \in \tilde{Z}$, we obtain

$$
\begin{aligned}
f(z) & \geq f(\bar{z})+\xi_{1}^{\top}(z-\bar{z})=f(\bar{z})-\xi_{2}^{\top}(z-\bar{z})-\xi_{3}^{\top}(z-\bar{z})-\xi_{4}^{\top}(z-\bar{z}) \\
& \geq f(\bar{z})-\xi_{2}^{\top}(z-\bar{z})-(-\mu)^{\top}(\lambda-\bar{\lambda})-\xi_{3}^{\top}(z-\bar{z})-\mu^{\top}(\lambda-\bar{\lambda})-(-v)^{\top}(0-0) \\
& \geq f(\bar{z})
\end{aligned}
$$

by definition of the subdifferential and the normal cone in the sense of convex analysis. This shows that $(\bar{z}, \bar{\lambda})$ is a global minimizer of $(\mathrm{Q})$, i.e., $\bar{z}$ is a global minimizer of $(\mathrm{P})$ due to Theorem $4 \cdot 3$.

\section{CONSEQUENCES FOR CERTAIN PROBLEM CLASSES}

In this section, we discuss some of the results obtained in Section 4 by means of the example problems introduced in Section 2.

\subsection{BILEVEL PROGRAMMING}

In this section, we take a look back at the bilevel programming problem (BPP) from Section 2.1. Throughout the section, the following additional standing assumption may hold.

Assumption 5.1. The lower level feasibility mapping $y \rightrightarrows g(y)-C$ is metrically subregular at all points $(y, 0)$ belonging to its graph.

Clearly, the requirements from Assumption 5.1 are inherent whenever a Robinson-type constraint qualification holds at all lower level feasible points. In case $C:=\mathbb{R}_{-}^{m}$, this amounts to validity of MFCQ at all points from $\Gamma$. However, in many situations, the metric subregularity assumption from Assumption 5.1 might be weaker.

Consulting Section 3.2 and [53, Theorem 6.14], we find that the pre-image formula

$$
\widehat{\mathcal{N}}_{\Gamma}(y)=\nabla g(y)^{\top} \widehat{\mathcal{N}}_{C}(g(y))=\nabla g(y)^{\top}\left[C^{\circ} \cap g(y)^{\perp}\right]
$$

holds for all $y \in \Gamma$. Thus, the associated intermediate mapping $K: \mathbb{R}^{n_{1}} \times \mathbb{R}^{n_{2}} \rightrightarrows \mathbb{R}^{m}$ is given by

$$
K(x, y)=\left\{\lambda \in C^{\circ} \mid \nabla_{y} j(x, y)+\nabla g(y)^{\top} \lambda=0, \lambda^{\top} g(y)=0\right\}
$$


which is nothing else but the so-called Lagrange multiplier mapping associated with (LL). Keeping Proposition 3.3 and the continuity of $\nabla_{y} j(\cdot, \cdot)$ in mind, $K$ is inner semicompact w.r.t. its domain everywhere. Thus, our Theorems 4.3 and 4.5 recover the results obtained in $[20,22]$ under validity of weaker constraint qualifications at the lower level. Indeed, we do not exploit the local boundedness of the intermediate mapping $K$, which was the key idea in the latter papers, but only its inner semicompactness. For that purpose, metric subregularity of the feasibility mapping is enough. Let us inspect the assertion of Corollary 4.6 in the light of the present setting. Therefore, we fix a point $((\bar{x}, \bar{y}), \bar{\lambda}) \in \operatorname{gph} K$. Relying on [14, Lemma 4.44, Proposition 4.47] and observing that $C$ is a cone, we obtain that $K$ is inner semicontinuous at $((\bar{x}, \bar{y}), \bar{\lambda})$ if the condition

$$
\nabla g(\bar{y})^{\top} \lambda=0, \lambda^{\top} g(\bar{y})=0, \lambda \in\left(C \cap \bar{\lambda}^{\perp}\right)^{\circ} \quad \Longrightarrow \quad \lambda=0
$$

is valid. Actually, this already implies $K(\bar{x}, \bar{y})=\{\bar{\lambda}\}$ and that $K$ is so-called locally upper Lipschitz continuous at $(\bar{x}, \bar{y})$, see [14, Section 2.3] for a definition. In general, (5.1) is more restrictive than validity of GMFCQ at $\bar{y}$. Noting that (5.1) depends on the multiplier $\bar{\lambda}$ and, thus, implicitly on the objective function $j$, this condition is not a constraint qualification in the narrower sense. In the setting of standard nonlinear programming, i.e., where $C:=\mathbb{R}_{-}^{m}$ holds, (5.1) is referred to as the strict Mangasarian-Fromovitz condition which is known to be the weakest condition implying uniqueness of Lagrange multipliers, see [55]. In the more general setting where $C$ is a polyhedral, convex cone, (5.1) is implied by the so-called non-degeneracy condition

$$
\nabla g(\bar{y})^{\top} \lambda=0, \lambda \in\left(C^{\circ} \cap g(\bar{y})^{\perp}-C^{\circ} \cap g(\bar{y})^{\perp}\right) \quad \Longrightarrow \quad \lambda=0
$$

which corresponds to the prominent linear independence constraint qualification (LICQ) in standard nonlinear programming. It can be distilled from [20, Theorem 3.2] that a bilevel optimization problem and its reformulated single-level counterpart, where lower level multipliers are treated as explicit variables, are equivalent w.r.t. local minimizers whenever LICQ is valid at each point which is feasible for the lower level problem. In this regard, the above observations provide another generalization of the results from [2o]. Let us also mention that if $C$ is polyhedral, we can actually obtain stronger properties of $K$ which correspond to inner semicontinuity and inner semicompactness but with linear rate of change (called inner calmness and inner calmness ${ }^{*}$, respectively, in $[6,9]$, where more details can be found).

Recalling the setting from (2.1), we have $H(z):=\nabla_{y} j(z)+\widehat{\mathcal{N}}_{\Gamma}(y)$ for all $z:=(x, y)$. In this regard, the implicit M-stationarity conditions of (BPP) at some feasible point $\bar{z}:=(\bar{x}, \bar{y})$ reduce to the existence of $v \in \mathbb{R}^{n_{2}}$ such that

$$
(0,0) \in \partial f(\bar{z})+\nabla_{y z}^{2} j(\bar{z})^{\top} v+\{0\} \times D^{*} \widehat{\mathcal{N}}_{\Gamma}\left(\bar{y},-\nabla_{y} j(\bar{z})\right)(v)+\mathcal{N}_{S}(\bar{x}) \times\{0\} .
$$

Some recent progress in the field of variational analysis even allows to calculate or estimate the appearing coderivative of the normal cone mapping $\widehat{\mathcal{N}}_{\Gamma}: \mathbb{R}^{n_{2}} \rightrightarrows \mathbb{R}^{n_{2}}$ in terms of initial data under suitable assumptions, see e.g. $[31,32,34]$. On the other hand, the explicit M-stationarity conditions of (BPP) reduce to the existence of $\bar{\lambda} \in K(\bar{z})$ and $v \in \mathbb{R}^{n_{2}}$ which satisfy

$$
\begin{aligned}
(0,0) \in \partial f(\bar{z})+\nabla_{y z}^{2} j(\bar{z})^{\top} v+\left\{\left(0, \sum_{i=1}^{m} \bar{\lambda}_{i} \nabla^{2} g_{i}(\bar{y}) v\right)\right\} & \\
& +\{0\} \times D^{*}\left(\widehat{\mathcal{N}}_{C} \circ g\right)(\bar{y}, \bar{\lambda})(\nabla g(\bar{y}) v)+\mathcal{N}_{S}(\bar{x}) \times\{0\},
\end{aligned}
$$

see statement (a) of Proposition 4.21. Above, $g_{1}, \ldots, g_{m}: \mathbb{R}^{n_{2}} \rightarrow \mathbb{R}$ denote the component functions of $g$. Naturally, this stationarity system might be deduced directly from the above system of implicit M-stationarity with the aid of the chain rule from Lemma 3.4. Consulting Proposition 3.3 once more, however, we see that the intermediate mapping $\widehat{K}$ from (4.3), which takes the form

$$
\widehat{K}((x, y), w)=\left\{\lambda \in C^{\circ} \mid-w+\nabla_{y} j(x, y)+\nabla g(y)^{\top} \lambda=0, \lambda^{\top} g(y)=0\right\},
$$


is also inner semicompact w.r.t. its domain at all points $((\bar{x}, \bar{y}), 0)$ that satisfy the relation $(\bar{x}, \bar{y}) \in \operatorname{dom} K$. In this regard, statement (a) of Proposition 4.21 guarantees that each implicitly M-stationary point of (BPP) is automatically explicitly M-stationary as well. This observation generalizes the classical results from [49].

Note that the coderivative of $\widehat{\mathcal{N}}_{C} \circ g$ might be computed or estimated using again a suitable chain rule while observing that the associated intermediate mapping from (3.2) is naturally inner semicontinuous w.r.t. its domain at each point of its graph by continuity of $g$. This way, one might be tempted to call the stationarity system

$$
\begin{aligned}
(0,0) \in \partial f(\bar{z})+\nabla_{y z}^{2} j(\bar{z})^{\top} v+\left\{\left(0, \sum_{i=1}^{m} \bar{\lambda}_{i} \nabla^{2} g_{i}(\bar{y}) v\right)\right\} \\
\quad+\{0\} \times \nabla g(\bar{y})^{\top} D^{*} \widehat{\mathcal{N}}_{C}(g(\bar{y}), \bar{\lambda})(\nabla g(\bar{y}) v)+\mathcal{N}_{S}(\bar{x}) \times\{0\}
\end{aligned}
$$

the fully explicit M-stationarity system of (BPP) (w.r.t. $\bar{\lambda}$ ). We want to point out that this system can be written equivalently as

$$
\begin{aligned}
& (0,0) \in \partial f(\bar{z})+\nabla_{y z}^{2} j(\bar{z})^{\top} v+\left\{\left(0, \sum_{i=1}^{m} \bar{\lambda}_{i} \nabla^{2} g_{i}(\bar{y}) v+\nabla g(\bar{y})^{\top} \mu\right)\right\}+\mathcal{N}_{S}(\bar{x}) \times\{0\} \\
& (\mu,-\nabla g(\bar{y}) v) \in \mathcal{N}_{\mathrm{gph} \widehat{\mathcal{N}}_{C}}(g(\bar{y}), \bar{\lambda}),
\end{aligned}
$$

corresponding to the M-stationarity system of the optimization problem

$$
\begin{aligned}
f(x, y) & \rightarrow \min _{x, y, \lambda} \\
x & \in S \\
\nabla_{y} j(x, y)+\nabla g(y)^{\top} \lambda & =0 \\
(g(y), \lambda) & \in \operatorname{gph} \widehat{\mathcal{N}}_{C} .
\end{aligned}
$$

Observing that $C$ is a convex cone, we have

$$
\operatorname{gph} \widehat{\mathcal{N}}_{C}=\left\{(a, b) \in C \times C^{\circ} \mid a^{\top} b=0\right\},
$$

and this motivates us to call (5.2) a generalized mathematical problem with complementarity constraints, see [29, Section 4] or [56]. Following the above arguments and keeping Section 3.2 in mind, we obtain the following result.

Corollary 5.2. Let $\bar{z}:=(\bar{x}, \bar{y}) \in \mathbb{R}^{n_{1}} \times \mathbb{R}^{n_{2}}$ be a local minimizer of (BPP) and assume that, for some $\bar{\lambda} \in K(\bar{z})$, the mapping

$$
(x, y, \lambda) \rightrightarrows\left(x-S, \nabla_{y} j(x, y)+\nabla g(y)^{\top} \lambda,(g(y), \lambda)-\operatorname{gph} \widehat{\mathcal{N}}_{C}\right)
$$

is metrically subregular at $((\bar{x}, \bar{y}, \bar{\lambda}),(0,0,(0,0)))$. Then $\bar{z}$ is fully explicitly M-stationary for (BPP) w.r.t. $\bar{\lambda}$.

Using the above terminology, we would like to mention that the authors in [1] discussed the implicit and fully explicit M-stationarity conditions of (BPP) in case $C:=\mathbb{R}_{-}^{m}$. They came up with refined conditions ensuring that the feasibility mapping from Corollary 5.2 is indeed metrically subregular at some reference point, see [1, Theorem 8]. From the viewpoint of applicability, the system of fully explicit M-stationarity might be the most useful one among the stated ones since the appearing coderivative of the normal cone mapping $\widehat{\mathcal{N}}_{C}$ is computable in some situations where the set $C$ is simple, see e.g. the proof of [24, Theorem 2] and [33, Theorem 2.12]. Let us mention that yet another M-stationarity-type system associated with (BPP) has been derived in the recent paper [36]. 


\subsection{EVALUATING WEAKLY EFFICIENT POINTS IN MULTICRITERIA OPTIMIZATION}

Let us consider (EMOP) under the assumptions from Section 2.2. Particularly, we consider problem (P) with the setting from (2.2). The associated intermediate mapping $K: \mathbb{R}^{n} \rightrightarrows \mathbb{R}^{m}$ reads as

$$
\forall z \in \mathbb{R}^{n}: \quad K(z)=\{\lambda \in \Delta \mid z \in \Psi(\lambda)\}
$$

The subsequent lemma provides essential foundations of our analysis.

Lemma 5.3. The mapping $G$ from (2.2) possesses a closed graph while $K$ from above is inner semicompact w.r.t. its domain everywhere.

Proof. First, we show that $G$ possesses a closed graph. Choose sequences $\left\{z_{k}\right\}_{k \in \mathbb{N}} \subset \mathbb{R}^{n},\left\{\lambda_{k}\right\}_{k \in \mathbb{N}} \subset \mathbb{R}^{m}$, and $\left\{w_{k}\right\}_{k \in \mathbb{N}} \subset \mathbb{R}^{n}$ such that $z_{k} \rightarrow z, \lambda_{k} \rightarrow \lambda$, and $w_{k} \rightarrow w$ for some $z \in \mathbb{R}^{n}, \lambda \in \mathbb{R}^{m}$, and $w \in \mathbb{R}^{n}$ are valid while $w_{k} \in G\left(z_{k}, \lambda_{k}\right)$ holds for all $k \in \mathbb{N}$. Particularly, we find $\left\{\lambda_{k}\right\}_{k \in \mathbb{N}} \subset \Delta$ and $z_{k}+w_{k} \in \Psi\left(\lambda_{k}\right)$, i.e.,

$$
\forall k \in \mathbb{N}, \forall z^{\prime} \in \Gamma: \quad \lambda_{k}^{\top} j\left(z_{k}+w_{k}\right) \leq \lambda_{k}^{\top} j\left(z^{\prime}\right)
$$

and $z_{k}+w_{k} \in \Gamma$ for all $k \in \mathbb{N}$. Taking the limit $k \rightarrow \infty$ and observing that $j$ is continuous while $\Gamma$ and $\Delta$ are closed, we infer $z+w \in \Gamma$ and $\lambda^{\top} j(z+w) \leq \lambda^{\top} j\left(z^{\prime}\right)$ for all $z^{\prime} \in \Gamma$. This yields $z+w \in \Psi(\lambda)$ which equals $w \in G(z, \lambda)$, i.e., $((z, \lambda), w) \in \operatorname{gph} G$. Particularly, gph $G$ is closed.

By definition, $F$ is locally bounded everywhere which is why the lemma's assertion regarding $K$ follows directly from Lemma 4.2.

Now, Theorems 4.3 and 4.5 can be used to infer the precise relationship between (EMOP) and its associated counterpart (Q) w.r.t. global and local minimizers, respectively. Related results can be obtained in the slightly more general context of semivectorial bilevel programming where the lower level decision maker has to solve a multiobjective optimization problem, see [23].

Let us now focus on optimality conditions for (EMOP). Clearly, the associated implicit M-stationarity conditions take the form

$$
0 \in \partial f(\bar{z})+\mathcal{N}_{\Gamma_{\mathrm{we}}}(\bar{z})
$$

for an arbitrary feasible point $\bar{z} \in \mathbb{R}^{n}$. However, these conditions are of limited practical use due to an essential lack of knowledge regarding the variational geometry of $\Gamma_{\mathrm{we}}$. On the other hand, invoking Proposition 4.21, we find that the explicit M-stationarity conditions of (EMOP) take the following form: there exist $\bar{\lambda} \in K(\bar{z})$ and $v \in \mathbb{R}^{n}$ such that

$$
v \in \partial f(\bar{z}), \quad 0 \in D^{*} \Psi(\bar{\lambda}, \bar{z})(v)+\mathcal{N}_{\Delta}(\bar{\lambda}) .
$$

Taking into account that

$$
\forall \lambda \in \Delta: \quad \Psi(\lambda)=\left\{z \in \mathbb{R}^{n} \mid 0 \in \nabla j(z)^{\top} \lambda+\widehat{\mathcal{N}}_{\Gamma}(z)\right\}
$$

holds, we refer to [25, 33] for a broader view on solution mappings of parametrized variational systems and estimates of their generalized derivatives. Particularly, in case where $j$ is twice continuously differentiable, we obtain the additional estimate

$$
D^{*} \Psi(\bar{\lambda}, \bar{z})(v) \subset\left\{\nabla j(\bar{z}) \mu \mid-v \in \sum_{i=1}^{m} \bar{\lambda}_{i} \nabla^{2} j_{i}(\bar{z}) \mu+D^{*} \widehat{\mathcal{N}}_{\Gamma}\left(\bar{z},-\nabla j(\bar{z})^{\top} \bar{\lambda}\right)(\mu)\right\}+\mathcal{N}_{\Delta}(\bar{\lambda})
$$

applying the pre-image rule to

$$
\operatorname{gph} \Psi=\left\{(\lambda, z) \mid\left(z,-\nabla j(z)^{\top} \lambda, \lambda\right) \in \operatorname{gph} \widehat{\mathcal{N}}_{\Gamma} \times \Delta\right\}
$$


which is possible whenever the feasibility mapping $(\lambda, z) \rightrightarrows\left(z,-\nabla j(z)^{\top} \lambda, \lambda\right)-\operatorname{gph} \widehat{\mathcal{N}}_{\Gamma} \times \Delta$ is metrically subregular at $((\bar{\lambda}, \bar{z}),(0,0,0))$, see Section 3.2. The coderivative of the normal cone mapping associated with $\Gamma$ can be estimated from above in several interesting situations covering the setting where $\Gamma$ is the pre-image of a closed and convex (polyhedral) set under a sufficiently smooth mapping, see the references mentioned in Section 5.1. Using a related approach, fully explicit necessary optimality condition for semivectorial bilevel optimization problems are derived in [59].

Observing that $F$ and $G$ are metrically subregular mappings in the present setting (2.2), metric subregularity of the mapping $\widetilde{\Sigma}: \mathbb{R}^{n} \times \mathbb{R}^{m} \rightrightarrows \mathbb{R}^{m} \times \mathbb{R}^{m+n}$ given by

$$
\forall z \in \mathbb{R}^{n}, \forall \lambda \in \mathbb{R}^{m}: \quad \widetilde{\Sigma}(z, \lambda):=(\lambda-\Delta) \times((\lambda, z)-\operatorname{gph} \Psi)
$$

at $((\bar{z}, \bar{\lambda}),(0,(0,0)))$ for some $\bar{\lambda} \in K(\bar{z})$ is already enough to obtain that the associated mapping $\mathcal{H}_{M}$ is metrically subregular at $((\bar{z}, \bar{\lambda}),(0,0))$, see Lemma 3.8. Note that $\widetilde{\Sigma}$ results from the mapping $\Sigma$ given in Lemma 3.8 by deleting the trivial factor $\mathbb{R}^{n}$ which does not influence the metric subregularity property. In the presence of this metric subregularity, $\bar{z}$ is an explicitly M-stationary point of (EMOP) provided $\bar{z}$ is a local minimizer of this program, see Proposition 4.11 and the arguments in Section 4.3.

\subsection{CARDINALITY-CONSTRAINED PROGRAMMING}

Finally, we investigate the setting of cardinality-constrained optimization from Section 2.3. In the context of (2.5), the mapping $K: \mathbb{R}^{n} \rightrightarrows \mathbb{R}^{n}$ is given by

$$
\forall z \in \mathbb{R}^{n}: \quad K(z)=\left\{\lambda \in[0,1]^{n} \mid \sum_{i \in I^{0}(z)} \lambda_{i} \geq n-\kappa, \forall i \in I^{ \pm}(z): \lambda_{i}=0\right\}
$$

where we used

$$
I^{ \pm}(z):=\left\{i \in\{1, \ldots, n\} \mid z_{i} \neq 0\right\}, \quad I^{0}(z):=\left\{i \in\{1, \ldots, n\} \mid z_{i}=0\right\}
$$

for arbitrary $z \in \mathbb{R}^{n}$. Obviously, $K$ is locally bounded and, thus, inner semicompact w.r.t. the set $\operatorname{dom} K=\left\{z \in \mathbb{R}^{n} \mid\|z\|_{0} \leq \kappa\right\}$ at all points of its domain. The images of $K$ are polytopes (i.e., bounded polyhedrons). Furthermore, $K(z)$ is a singleton if and only if $z$ satisfies $\|z\|_{0}=\kappa$. In this regard, Theorem 4.3 recovers [17, Theorem 3.2]. On the other hand, the results [17, Proposition 3.5, Theorem 3.6] are consequences of Theorem 4.5. However, our result even applies to settings where $\|z\|_{0}<\kappa$ is valid, and, thus, clarifies the situation in [17, Examples 1 and 2].

Let us mention first that due to the arguments from Section 2.3, the mapping $H$ takes the form $z \rightrightarrows D_{\kappa}-z$ in the situation at hand, see (2.3), and we note that it is polyhedral by nature of $D_{\kappa}$ (since $D_{\kappa}$ is the union of finitely many convex, polyhedral sets). The implicit M-stationarity conditions of (CCMP) w.r.t. a feasible point $\bar{z} \in \mathbb{R}^{n}$ are given by

$$
0 \in \partial f(\bar{z})+\mathcal{N}_{D_{\kappa}}(\bar{z})+\mathcal{N}_{M}(\bar{z}) .
$$

By means of the formula

$$
\mathcal{N}_{D_{\kappa}}(\bar{z})=\left\{v \in \mathbb{R}^{n} \mid\|v\|_{0} \leq n-\kappa, \forall i \in I^{ \pm}(\bar{z}): v_{i}=0\right\},
$$

which can be distilled from [50, Lemma 2.3], we obtain a reasonable optimality condition as soon as the variational structure of $M$ is nice enough. Let us point out that whenever $M$ is the union of finitely many convex polyhedral sets, then each local minimizer of the associated problem (CCMP) is implicitly M-stationary by Remark 4.12 since the feasibility mapping $z \rightrightarrows\left(z-D_{\kappa}\right) \times(z-M)$ is polyhedral in this situation and, thus, metrically subregular at all points of its graph. In case where the variational geometry of $M$ is more difficult, metric subregularity of this feasibility mapping at $(\bar{z},(0,0))$ is still enough to infer implicit M-stationarity whenever $\bar{z}$ is a local minimizer of (CCMP). In case where 
$M:=\left\{z \in \mathbb{R}^{n} \mid \Phi(z) \in \Omega\right\}$ holds for a continuously differentiable mapping $\Phi: \mathbb{R}^{n} \rightarrow \mathbb{R}^{p}$ and a closed set $\Omega \subset \mathbb{R}^{p}$, the associated Mordukhovich criterion, which ensures metric regularity of the feasibility mapping and validity of the pre-image rule for the estimation of the limiting normal cone to $D_{\kappa} \cap M$ at $\bar{z}$, takes the form

$$
\left\|\nabla \Phi(\bar{z})^{\top} \eta\right\|_{0} \leq n-\kappa, \eta \in \mathcal{N}_{\Omega}(\Phi(\bar{z})), \forall i \in I^{ \pm}(\bar{z}):\left(\nabla \Phi(\bar{z})^{\top} \eta\right)_{i}=0 \quad \Longrightarrow \quad \eta=0 .
$$

As we pointed out in Section 4.3, the fuzzy and explicit M-stationarity conditions of (CCMP) which follow from the setting (2.5) coincide. Performing some calculations and keeping statement (a) of Proposition 4.21 in mind, these explicit M-stationarity conditions take the following form at a reference point $\bar{z}$ : there exists some $\bar{\lambda} \in K(\bar{z})$ such that

$$
0 \in \partial f(\bar{z})+\left\{v \in \mathbb{R}^{n} \mid \forall i \in I^{ \pm}(\bar{z}): v_{i}=0\right\}+\mathcal{N}_{M}(\bar{z}) .
$$

We note that this condition does not depend on $\bar{\lambda}$ at all. It corresponds to the M-stationarity notion discussed in $[16,17,18]$. At the first glance, we observe that the implicit M-stationarity condition is more restrictive than its explicit counterpart. In light of statement (a) of Proposition 4.21, this is not surprising since the intermediate mapping $\widehat{K}$ associated with the present setting is inner semicompact w.r.t. its domain at each point of the latter. Recall that the explicit M-stationarity system does not depend on the precise choice of the implicit variable from $K(\bar{z})$, i.e., implicit M-stationarity of $\bar{z}$ implies explicit M-stationarity w.r.t. all implicit variables from $K(\bar{z})$. On the other hand, let us mention that in case of $\bar{z}:=0$ being feasible to (CCMP), it is always an explicitly M-stationary point of (CCMP) w.r.t. each implicit variable from $K(\bar{z})$ while this point is not necessarily implicitly M-stationary.

\section{CONCLUSIONS}

The essential message of this paper says that whenever optimization problems with implicit variables are under consideration, it is better to leave them implicit as long as possible. In many practically relevant situations like cardinality-constrained programming, see Section 5.3, this procedure leads to more restrictive necessary optimality conditions which hold under less restrictive constraint qualifications (in a certain sense). Furthermore, the implicit formulation avoids the appearance of artificial local minimizers, and this might be beneficial not only from a theoretical but also from a numerical point of view. It is, thus, always desirable to explore the inherent problem structure of the original problem instead of making its implicit variables explicit for a non-negligible price. Exemplary, let us mention that a convincing variational description of the weakly efficient set of a multiobjective optimization problem which avoids the use of scalarization variables is likely to enhance the results from Section 5.2.

\section{REFERENCES}

[1] L. Adam, R. Henrion, and J. Outrata, On M-stationarity conditions in MPECs and the associated qualification conditions, Mathematical Programming 168 (2018), 229-259, doi:10.1007/ s10107-017-1146-3.

[2] D. Aussel and A. Svensson, Is pessimistic bilevel programming a special case of a mathematical program with complementarity constraints?, Journal of Optimization Theory and Applications 181 (2019), 504-520, doi:10.1007/s10957-018-01467-7.

[3] K. Bai, J.J. Ye, and J. Zhang, Directional quasi-/pseudo-normality as sufficient conditions for metric subregularity, SIAM fournal on Optimization 29 (2019), 2625-2649, doi:10.1137/18m1232498. 
[4] J. F. Bard, Practical Bilevel Optimization: Algorithms and Applications, Kluwer Academic, Dordrecht, 1998.

[5] A. Beck and N. Hallak, On the minimization over sparse symmetric sets: projections, optimality conditions, and algorithms, Mathematics of Operations Research 41 (2016), 196-223, doi:10.1287/ moor.2015.0722.

[6] M. Benko, On inner calmness*, generalized calculus, and derivatives of the normal-cone mapping, fournal of Nonsmooth Analysis and Optimization 2 (2021), 5881, doi:10.46298/jnsao-2021-5881.

[7] M. Benko, M. Červinka, and T. Hoheisel, Sufficient conditions for metric subregularity of constraint systems with applications to disjunctive and ortho-disjunctive programs, Set-Valued and Variational Analysis (2021), 1-35, doi:10.1007/s11228-020-00569-7.

[8] M. Benko, H. Gfrerer, and J. V. Outrata, Calculus for directional limiting normal cones and subdifferentials, Set-Valued and Variational Analysis 27 (2019), 713-745, doi:10.1007/s11228-018-0492-5.

[9] M. Benko and P. Mehlitz, Calmness and calculus: two basic patterns, Set-Valued and Variational Analysis (2021), 1-37, doi:10.1007/s11228-021-00589-x.

[10] H. P. Benson, An algorithm for optimizing over the weakly-efficient set, European fournal of Operational Research 25 (1986), 192-199, doi:10.1016/0377-2217(86)90085-8.

[11] D. Bienstock, Computational study of a family of mixed-integer quadratic programming problems, Mathematical Programming 74 (1996), 121-140, doi:10.1007/bfo2592208.

[12] S. Bolintineanu, Necessary conditions for nonlinear suboptimization over the weakly-efficient set, Journal of Optimization Theory and Applications 78 (1993), 579-598, doi:10.1007/bfoog39883.

[13] S. Bolintineanu, Optimality conditions for minimization over the (weakly or properly) efficient set, fournal of Mathematical Analysis and Applications 173 (1993), 523-541, doi:10.1006/jmaa.1993.1085.

[14] J.F. Bonnans and A. Shapiro, Perturbation Analysis of Optimization Problems, Springer, New York, 2000.

[15] R. I. Boţ, E. R. Csetnek, and E. Nagy, Solving systems of monotone inclusions via primal-dual splitting techniques, Taiwanese fournal of Mathematics 17 (2013), 1983-2009, doi:10.11650/tjm.17. 2013.3087.

[16] M. Bucher and A. Schwartz, Second-order optimality conditions and improved convergence results for regularization methods for cardinality-constrained optimization problems, fournal of Optimization Theory and Applications 178 (2018), 383-410, doi:10.1007/s10957-018-1320-7.

[17] O. P. Burdakov, C. Kanzow, and A. Schwartz, Mathematical programs with cardinality constraints: reformulation by complementarity-type conditions and a regularization method, SIAM fournal on Optimization 26 (2016), 397-425, doi:10.1137/140978077.

[18] M. Červinka, C. Kanzow, and A. Schwartz, Constraint qualifications and optimality conditions for optimization problems with cardinality constraints, Mathematical Programming 160 (2016), 353-377, doi:10.1007/s10107-016-0986-6.

[19] S. Dempe, Foundations of Bilevel Programming, Kluwer, Dordrecht, 2002. 
[20] S. Dempe and J. Dutta, Is bilevel programming a special case of a mathematical program with complementarity constraints?, Mathematical Programming 131 (2012), 37-48, doi:10.1007/ s10107-010-0342-1.

[21] S. Dempe, V. Kalashnikov, G. Pérez-Valdéz, and N. Kalashnykova, Bilevel Programming Problems - Theory, Algorithms and Applications to Energy Networks, Springer, Berlin, 2015.

[22] S. Dempe, F. Mefo Kue, and P. Mehlitz, Optimality conditions for special semidefinite bilevel optimization problems, SIAM Journal on Optimization 28 (2018), 1564-1587, doi:10.1137/16m1099303.

[23] S. Dempe and P. Mehlitz, Semivectorial bilevel programming versus scalar bilevel programming, Optimization 69 (2020), 657-679, doi:10.1080/02331934.2019.1625900.

[24] A.L. Dontchev and R. T. Rockafellar, Characterizations of strong regularity for variational inequalities over polyhedral convex sets, SIAM fournal on Optimization 6 (1996), 1087-1105, doi:10.1137/s1052623495284029.

[25] A. L. Dontchev and R. T. Rockafellar, Implicit Functions and Solution Mappings, Springer, Heidelberg, 2014.

[26] M. Ehrgott, Multicriteria Optimization, Springer, Berlin, 2005.

[27] M.J. Fabian, R. Henrion, A. Y. Kruger, and J.V. Outrata, Error bounds: necessary and sufficient conditions, Set-Valued and Variational Analysis 18 (2010), 121-149, doi:10.1007/s11228-010-0133-0.

[28] M.L. Flegel, C. Kanzow, and J.V. Outrata, Optimality conditions for disjunctive programs with application to mathematical programs with equilibrium constraints, Set-Valued Analysis 15 (2007), 139-162, doi:10.1007/s11228-006-0033-5.

[29] S. Franke, P. Mehlitz, and M. Pilecka, Optimality conditions for the simple convex bilevel programming problem in Banach spaces, Optimization 67 (2018), 237-268, doi:10.1080/02331934.2017. 1394296.

[30] H. Gfrerer and D. Klatte, Lipschitz and Hölder stability of optimization problems and generalized equations, Mathematical Programming 158 (2016), 35-75, doi:10.1007/s10107-015-0914-1.

[31] H. Gfrerer and J.V. Outrata, On computation of generalized derivatives of the normal-cone mapping and their applications, Mathematics of Operations Research 41 (2016), 1535-1556, doi: 10.1287/moor.2016.0789.

[32] H. Gfrerer and J.V. Outrata, On computation of limiting coderivatives of the normal-cone mapping to inequality systems and their applications, Optimization 65 (2016), 671-70o, doi: 10.1080/02331934.2015.1066372.

[33] H. Gfrerer and J.V. Outrata, On Lipschitzian properties of implicit multifunctions, SIAM fournal on Optimization 26 (2016), 2160-2189, doi:10.1137/15m1052299.

[34] H. Gfrerer and J. V. Outrata, On the Aubin property of a class of parameterized variational systems, Mathematical Methods of Operations Research 86 (2017), 443-467, doi:10.1007/s00186-017-0596-y.

[35] H. Gfrerer and J. V. Outrata, On a semismooth* Newton method for solving generalized equations, SIAM fournal on Optimization 31 (2021), 489-517, doi:10.1137/19m1257408. 
[36] H. Gfrerer and J.J. Ye, New sharp necessary optimality conditions for mathematical programs with equilibrium constraints, Set-Valued and Variational Analysis 28 (2020), 395-426, doi:10.1007/ s11228-019-00519-y.

[37] R. Henrion, A. Jourani, and J. V. Outrata, On the calmness of a class of multifunctions, SIAM fournal on Optimization 13 (2002), 603-618, doi:10.1137/s1052623401395553.

[38] R. Henrion and J. V. Outrata, Calmness of constraint systems with applications, Mathematical Programming 104 (2005), 437-464, doi:10.1007/s10107-005-0623-2.

[39] R. Horst and N. V. Thoai, Maximizing a concave function over the efficient or weakly-efficient set, European fournal of Operational Research 117 (1999), 239-252, doi:10.1016/so377-2217(98)oo230-6.

[40] A.D. Ioffe and J.V. Outrata, On metric and calmness qualification conditions in subdifferential calculus, Set-Valued Analysis 16 (2008), 199-227, doi:10.1007/s11228-008-0076-х.

[41] J. Jahn, Vector Optimization, Springer, Berlin, 2004.

[42] D. Klatte and B. Kummer, Constrained minima and Lipschitzian penalties in metric spaces, SIAM fournal on Optimization 13 (2002), 619-633, doi:10.1137/s105262340139625x.

[43] L. Lampariello, S. Sagratella, and O. Stein, The standard pessimistic bilevel problem, SIAM fournal on Optimization 29 (2019), 1634-1656, doi:10.1137/18m119759x.

[44] P. Mehlitz, A comparison of solution approaches for the numerical treatment of or-constrained optimization problems, Computational Optimization and Applications 76 (2020), 233-275, doi: 10.1007/s10589-020-00169-z.

[45] P. Mehlitz, On the linear independence constraint qualification in disjunctive programming, Optimization 69 (2020), 2241-2277, doi:10.1080/02331934.2019.1679811.

[46] P. Mehlitz, Stationarity conditions and constraint qualifications for mathematical programs with switching constraints, Mathematical Programming 181 (2020), 149-186, doi:10.1007/ s10107-019-01380-5.

[47] P. Mehlitz and A. B. Zemkoho, Sufficient optimality conditions in bilevel programming, Mathematics of Operations Research (2021), 1-26, doi:10.1287/moor.2021.1122.

[48] B. S. Mordukhovich, Variational Analysis and Applications, Springer, Cham, 2018.

[49] B. S. Mordukhovich and J. V. Outrata, Coderivative analysis of quasi-variational inequalities with applications to stability and optimization, SIAM fournal on Optimization 18 (2007), 389-412, doi:10.1137/060665609.

[50] L. Pan, N. Xiu, and J. Fan, Optimality conditions for sparse nonlinear programming, Science China Mathematics 6o (2017), 759-776, doi:10.1007/s11425-016-9010-x.

[51] L. L. Pan, N. H. Xiu, and S. L. Zhou, On solutions of sparsity constrained optimization, fournal of the Operations Research Society of China 3 (2015), 421-439, doi:10.1007/s40305-015-0101-3.

[52] S. M. Robinson, Some continuity properties of polyhedral multifunctions, in Mathematical Programming at Oberwolfach, H. König, B. Korte, and K. Ritter (eds.), Springer, Berlin, 1981, 206-214.

[53] R. T. Rockafellar and R. J. B. Wets, Variational Analysis, volume 317 of Grundlehren der mathematischen Wissenschaften, Springer, Berlin, 1998. 
[54] K. Shimizu, Y. Ishizuka, and J. F. Bard, Nondifferentiable and Two-level Mathematical Programming, Kluwer Academic, Dordrecht, 1997.

[55] G. Wachsmuth, On LICQ and the uniqueness of Lagrange multipliers, Operations Research Letters 41 (2013), 78 - 8o, doi:10.1016/j.orl.2012.11.009.

[56] G. Wachsmuth, Mathematical programs with complementarity constraints in Banach spaces, Journal of Optimization Theory and Applications 166 (2015), 480-507, doi:10.1007/s10957-014-0695-3.

[57] J.J. Ye and X. Y. Ye, Necessary optimality conditions for optimization problems with variational inequality constraints, Mathematics of Operations Research 22 (1997), 977-997, doi:10.1287/moor.22. 4.977.

[58] J.J. Ye and D. Zhu, New necessary optimality conditions for bilevel programs by combining the MPEC and value function approaches, SIAM fournal on Optimization 20 (2010), 1885-1905, doi:10.1137/080725088.

[59] A. B. Zemkoho, Solving ill-posed bilevel programs, Set-Valued and Variational Analysis 24 (2016), 423-448, doi:10.1007/s11228-016-0371-x.

[6o] X. Y. Zheng and K. F. Ng, Metric subregularity and calmness for nonconvex generalized equations in Banach spaces, SIAM fournal on Optimization 20 (2010), 2119-2136, doi:10.1137/090772174. 